La población inactiva en Colombia y sus características regionales. Un análisis desde modelos de descomposición microfactual

Jorge Alberto Castro Puello, José Guillermo Ariza Estévez y Armando Ardila Delgado 
Jorge Alberto Castro Puello, José Guillermo Ariza Estévezy Armando Ardila Delgado

\section{La población inactiva en Colombia y sus características regionales. Un análisis desde modelos de descomposición microfactual}

Resumen: Este articulo trata de explicar las diferencias en las tasas de inactividad laboral entre cada departamento de Colombia y el resto del pais. Se descompone este diferencial distinguiendo entre discrepancias en características observables —como distribución etaria, porcentaje de bombres y mujeres, ingresos, empleabilidad, etc.) y diferencias en características no observables (cultura, tradiciones, etcétera- Para esto, se utiliza un modelo de descomposición microfactorial para modelos no lineales aplicado a un modelo probit sobre la decisión de estar o no inactivo laboralmente. Se encuentran cuatro subgrupos de departamentos en función del factor influye más en la definición de su participación laboral. Se encontró que es más frecuente encontrar departamentos con mayor tasa de inactividad atribuida a los factores no observables.

Palabras clave: participación laboral, mercado laboral, descomposición microfactorial, economía regional, Probit.

Clasificación JEL: C13, J01, R10.

The Inactive Population in Colombia and its Regional Characteristics. An Analysis from Micro Factorial Decomposition Models

Abstract: This document tries to explain the differences in labor inactivity rates between each department of Colombia and the rest of the country. This differential is broken down by distinguishing between discrepancies in observable characteristics (i.e., age distribution, percentage of men and women, income, employability, etc.) and differences in unobservable characteristics (culture, traditions, etc.). For this we use a micro factorial decomposition model for nonlinear models applied to a probit model on the decision of whether or not to be inactive at work. There are 4 subgroups of departments depending on which factor most influences the definition of their labor participation. It was found that it is more common to find departments with a higher rate of inactivity attributed to unobservable factors.

Keywords: labor participation rate, labor market, micro factorial decomposition, regional economies, probit.

https://doi.org/10.17533/udea.le.n96a343954

\section{(cc) BY-NC-SA}

Este artículo y sus anexos se distribuyen por la revista Lecturas de Economía bajo los términos de la Licencia Creative Commons Atribución-NoComercial-CompartirIgual 4.0. https://creativecommons.org/licenses/by-nc-sa/4.0/ 
La population inactive en Colombie et ses caractéristiques régionales. Une analyse à partir de modèles de décomposition microfactuelle

Résumé: Cet article tente d'expliquer les différences dans les taux d'inactivité au travail entre chaque département de la Colombie et le reste du pays. Cet écart est décomposé en faisant la distinction entre les écarts dans les caractéristiques observables (comme la répartition par tranche d'âge, le pourcentage d'hommes et de femmes, le revenu, l'employabilité, etc.) et les différences dans les caractéristiques non observables (culture, traditions, etc.). Pour cela, est utilisé un modèle de décomposition microfactorielle pour les modèles non linéaires appliqués à un modèle probit sur la décision d'être inactif professionnellement ou de ne pas l'être. On a trowvé quatre sous-groupes de départements en fonction du facteur qui influence le plus dans la définition de leur participation au marché du travail. Il a été constaté qu'il est plus fréquent de trouver des départements ayant un taux d'inactivité plus élevé attribué à des facteurs non observables.

Mots clés: participation à la main-d'auvre, marché du travail, décomposition des microfacteurs, économie régionale, Probit.

Cómo citar / How to cite this item:

Castro-Puello, J. A., Ariza-Estévez, J. G., \& Ardila-Delgado, A. (2022). La población inactiva en Colombia y sus características regionales. Un análisis desde modelos de descomposición microfactual. Lecturas de Economía, 96, 31-70.

https://doi.org/10.17533/udea.le.n96a343954 


\title{
La población inactiva en Colombia y sus características regionales. Un análisis desde modelos de descomposición microfactual
}

\author{
Jorge Alberto Castro Puello $\oplus^{\mathrm{a}}$, José Guillermo Ariza Estévez $\oplus^{\mathrm{b}}$ y \\ Armando Ardila Delgado ${ }^{\mathrm{C}}$
}

Introducción. -I. Contexto. -II. Revisión de literatura. -III. Metodología y datos. -IV. Estimación. -V. Descomposición a nivel departamental. -VI. Discusión. -Conclusiones. -Referencias.

Primera versión recibida el 25 de septiembre de 2020; versión final aceptada el 20 de junio de 2021

\section{Introducción}

En este artículo se busca explicar el porqué de las diferencias estructurales en la población inactiva entre diferentes departamentos de Colombia, distinguiendo entre aspectos atribuibles a características sociodemográficas - como edad, promedio de la población, número de mujeres, ingresos medios, tamaño del hogar, etcétera- y aquellos que son producto de factores no observables - como cultura, tradiciones, idiosincrasia, valores, entre otros-

El estudio y análisis del mercado laboral se concentra, principalmente, en tres poblaciones: población ocupada, individuos desempleados y personas inactivas. Esta última, aunque no suele ser el foco de atención, permite complementar la caracterización estructural de los hogares y específicamente da una idea sobre escenarios futuros a los que podría verse expuesta la economía, con mayor o menor desempleo, productividad entre otros.

a Jorge Alberto Castro Puello: profesor de cátedra de la Universidad de los Andes, Facultad de Economía, Bogotá, Colombia. Dirección electrónica: ja.castro@uniandes.edu.co https://orcid.org/0000-0001-9949-3455

b José Guillermo Ariza Estévez: profesor de cátedra de la Universidad de los Andes, Facultad de Economía, Bogotá, Colombia. Dirección electrónica: jo-ariza@uniandes.edu.co https://orcid.org/0000-0002-2169-5839

c Armando Ardila Delgado: profesor de cátedra de la Universidad del Rosario, Facultad de Economía, Bogotá, Colombia. Dirección electrónica: armando.ardila@urosario.edu.co https://orcid.org/0000-0002-3678-0200 
Para ejemplificar esto, imagínese el lector un país con una tasa de ocupación alta, bajo nivel de desempleo y pocos inactivos. Esta situación puede ser buena o mala en función de la población inactiva. Por ejemplo, suponga que los jóvenes dejan de educarse para ingresar al mercado laboral por lo que la participación laboral será alta, sin embargo, pocas personas educándose pueden ser un problema a futuro. No obstante, la forma en la que se afronta esta situación es diferente sí este fenómeno se da porque hay muy pocos jóvenes en la zona o debido a que estos tienden con mayor frecuencia a buscar trabajo a temprana edad. El presente artículo aborda esta problemática exponiendo las diferencias estructurales en población inactiva entre los distintos departamentos y regiones de Colombia.

La inactividad suele ser representada por medio de su contraparte, es decir por la actividad laboral medida por la tasa global de participación (TGP). Esta representa el porcentaje de individuos en edad para trabajar que participan del mercado laboral ya sea trabajando o buscando trabajo. Este indicador refleja el tamaño relativo de la fuerza de trabajo y da indicios de la cantidad de población que se encuentra excluida del mercado laboral ya sea por edad, o incapacidad — pensión, discapacidad- o por decisión estudiantes, personas en oficios del hogar, etcétera-.

Por sí solo, la TGP no permite observar si el mercado laboral de un país es saludable o no. Es de notar que, una tasa de participación baja puede ser algo peligrosa si la mayoría de la población inactiva se encuentra en estado de discapacidad, o — en contraste — si está constituida por individuos en proceso de formación, puede conllevar a una mayor productividad en el futuro.

En la literatura económica es usual el estudio de los determinantes de la participación solo como parte de un proceso para explicar las diferencias salariales entre individuos. No obstante, las diferencias estructurales pueden ser un problema en sí mismas y, por ende, requerir de una mayor atención.

Estas diferencias estructurales pueden deberse a dos causas: la primera a factores observables asociados a la composición de la población y la segunda a no observables y relacionados con una serie de elementos que se pueden catalogar como culturales e idiosincráticos, propios y característicos de cada región. Un ejemplo de los primeros — asociados a diferencias en la 
estructura de la población-, se presenta cuando un subgrupo de individuos que es potencialmente inactivo se encuentra sobre representado, como poblaciones mayoritariamente jóvenes con una mayor propensión a estudiar o en poblaciones con participaciones importantes de grupos etarios mayores de 60 años - población adulta-tercera edad—, inactivos por su condición de pensionados u otras situaciones — como algún tipo incapacidad-.

Por el lado de los factores culturales e idiosincráticos se tiene aquellas poblaciones idénticas en su estructura poblacional, pero que - por elementos o determinantes culturales o idiosincráticos - hacen que un mismo subgrupo sea más o menos propenso a ser inactivo. Ejemplo de esto puede ser un grupo poblacional donde por tradición, las mujeres están más vinculadas a oficios del hogar u otros donde se incentiva más a los jóvenes a trabajar antes que estudiar.

Este artículo busca explicar las diferencias existentes en la población inactiva entre los distintos departamentos de Colombia, descomponiéndola en diferencias observables (género, edad, ingresos, etcétera) y factores no observables (idiosincrasia, creencias, cultura, tradiciones, entre otros). Para esto, se hace uso del método de descomposición microfactorial, que permite distinguir respuestas diferenciales en la probabilidad de participar en el mercado laboral ante las mismas características. La hipótesis por evaluar es que las diferencias regionales en dicho indicador no pueden atribuirse únicamente a factores observables.

Es de resaltar que el ejercicio como novedad, ajusta y aplica la técnica de descomposición para modelos no lineales. En síntesis, bajo esta aproximación se logra comparar dos poblaciones con diferencias en una variable - en nuestro caso, el porcentaje de inactivos-, consiguiendo dos estimaciones: la primera, que indica cuál sería el valor que tomaría esta variable si se intercambiaran las poblaciones manteniendo los factores no observables en cada grupo (departamento); y la segunda que expone cuál sería el valor de este indicador al mantenerse las poblaciones, pero intercambiando las características no observables.

El principal cambio con respecto a los métodos lineales se encuentra en que el efecto sobre la variable de interés cambia dependiendo del punto en el que se encuentran las otras variables. Para corregir esto se utiliza el 
método de Sinning et al. (2008), donde se toman los valores esperados, condicionandolos en las características observables y consiguiendose con esto una mejor aproximación del resultado tradicional.

Como principal resultado, se categoriza cada departamento en funcion de qué factores son mas relevantes dando una herramienta para el uso de políticas diferenciales para el mercado laboral colombiano.

El artículo está compuesto por una seccion de contexto donde se muestra las diferencias en la tasa de inactividad en Colombia. Posterior a esto, se presenta una revision de literatura continuando una descripcion de los datos y las variables a utilizar en el analisis. Luego, se incluye una sección donde se presentan las características observables entre regiones. Después, se expone el modelo y la metodologia a utilizar y se presentan los resultados empiricos observados. El artículo termina con una seccion de discusion y las principales conclusiones.

\section{Contexto}

Este estudio presta atención al caso colombiano, en donde la convergencia entre una población heterogénea, características geográficas diversas y la mezcla de estos componentes, han dado origen a múltiples regiones y lecturas de las diferentes variables. A continuación, se presentan algunos indicadores y variables relacionados con el mercado laboral.

Como se puede ver en la Figura 1, para el caso colombiano la tasa global de participación se ha mantenido estable alrededor de un 64,2\% desde el año 2011. En la Figura 2 se aprecia como a nivel departamental se encuentran tendencias divergentes con diferencias estructurales en la magnitud media de su población inactiva, estas pueden ser explicadas por las dinámicas económicas locales.

Adicionalmente, se logra identificar departamentos con niveles altos de inactividad — promediando el $40 \%$ o más-como lo son Atlántico, Bolívar, Boyacá, Caldas, Caquetá, Cesar y Magdalena o el caso del Choco que mantiene altos y crecientes niveles. Contrastando con tasas de inactividad relativamente bajas como las reportadas por Bogotá, Cundinamarca, Nariño, Santander, Tolima y Valle del Cauca. 
Figura 1. Tasa global de participación en Colombia 2011-2019

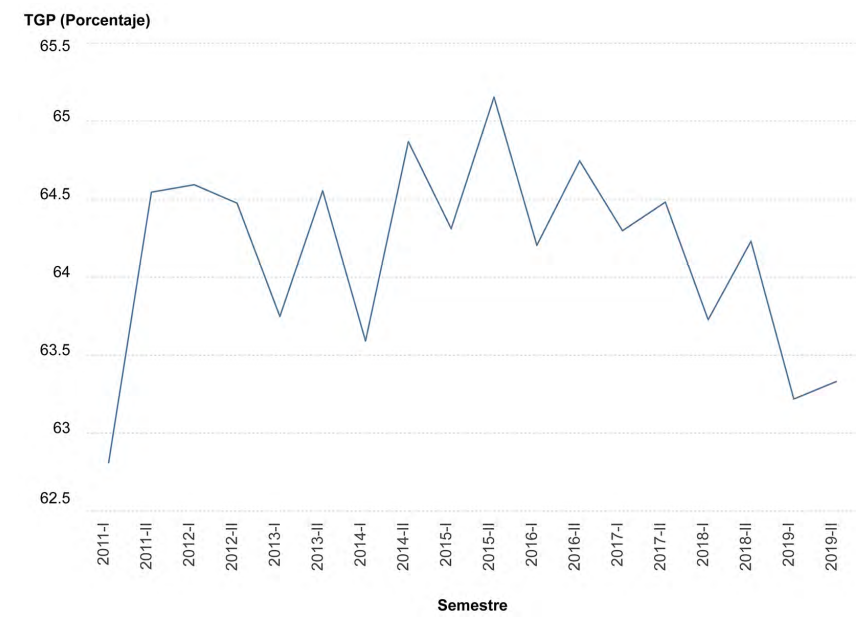

Fuente: elaboración propia a partir de DANE (s. f.).

Figura 2. Comportamiento población inactiva como porcentaje de la población en edad para trabajar desde 2005 hasta 2017 por departamentos

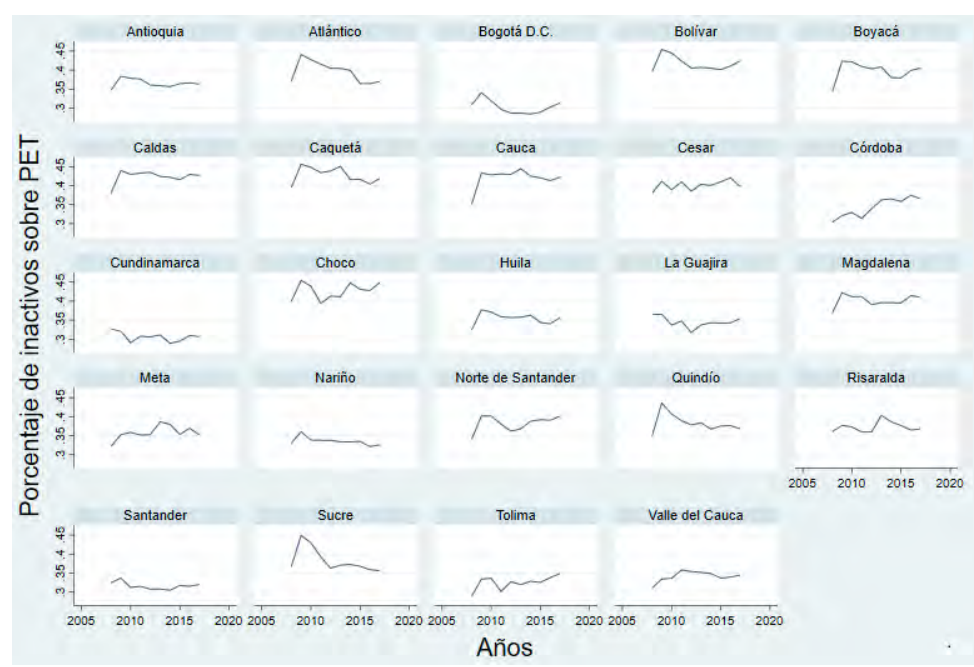

Fuente: elaboración propia a partir de DANE (s. f.). 
Castro Puello, Ariza Estévez y Ardila Delgado: La población inactiva en Colombia...

\section{Revisión de literatura}

La literatura sobre participación laboral, o inactividad laboral, se ha concentrado principalmente en la estimación como método para reducir el sesgo de selección en las aproximaciones salariales de población empleada, a partir del trabajo seminal de Heckman (1979). Esta metodología ha sido usual en estas estimaciones para diversos mercados (Dueñas et al., 2014; Uribe, 2006).

De forma específica, se han estudiado los determinantes de la participación laboral para subgrupos poblacionales como mujeres casadas (Gunderson, 1977; Karaoglan \& Okten, 2015; Mroz, 1987; Spencer, 1973), o personas de la tercera edad (Benitez \& Heiland, 1999; O’Brien, 2011). En Colombia se han estimado los determinantes de la participación laboral, teniendo como principal objetivo explicar la alta actividad laboral de la ciudad de Ibagué (Aldana \& Arango, 2007).

Los determinantes utilizados frecuentemente en la literatura incluyen características socioeconómicas como edad, estado civil y género, mientras que otras variables son usadas para medir factores como dependencia económica en el hogar o el nivel de ingresos (Aldana \& Arango, 2007; López, 2001; Santa María \& Rojas, 2001; Tenjo \& Ribero, 1998). De manera relacionada se ha estudiado la existencia de efectos como Added-Worker Effect y Discouraged-Worker Effect (Dagsvik et al., 2013).

En la literatura económica enfocada en mercado laboral se han desarrollado metodologías para el estudio en múltiples diferenciales -o gaps - como el gap salarial entre hombres y mujeres (Dueñas et al., 2014; Ospino et al., 2010). La herramienta más usada es la descomposición microfactorial a la Blinder-Oaxaca (Blinder, 1973; Oaxaca, 1973). Este método fue aplicado para determinar si la distancia en los salarios observados entre hombres y mujeres era producto de diferencias en características como educacion, experiencia u otros factores valorados por el mercado laboral o, si era causa de discriminacion hacia la mujer, evidenciada en que incluso reportando las mismas características observables que los hombres, estas tendrían salarios inferiores en promedio. 
Como recurso, ha sido ampliamente usado para descomponer estos diferenciales en los salarios, no obstante, no se ha explorado en la participación laboral. Entre los principales limitantes se encuentra que la metodología BlinderOaxaca se aplica para modelos lineales, mientras que los modelos no lineales son usualmente usados para estimar la participacion laboral — por ejemplo, modelos Probit y Logit- (Gunderson, 1977; Spencer, 1973).

Otras metodologías se han utilizado para descomponer el diferencial salarial entre géneros, como quantile regression (Machado \& Mata, 2005), o en otros casos se han tratado de incluir nuevas variables como resultados de pruebas estandarizadas para evaluar el canal por el cual se genera este diferencial (Meller et al., 2011).

En cuanto a métodos de descomposición microfactorial para modelos no lineales, se tiene el propuesto por Sinning et al. (2008), donde permiten aplicar la metodología Blinder-Oaxaca partiendo de la media condicionada de la variable dependiente y las medias condicionadas evaluadas con los parámetros de los grupos a comparar.

En este artículo se realiza una estimación de los determinantes de la inactividad laboral para los departamentos de Colombia por medio de un modelo Probit, junto a una descomposición para modelos no lineales (Sinning et al., 2008).

\section{Metodologia y datos}

En cuanto a los datos utilizados, el análisis toma información individualizada sobre personas en edad para trabajar en Colombia por departamento. La fuente utilizada es la Gran Encuesta Integrada de Hogares (GEIH) del Departamento Administrativo Nacional de Estadística (DANE), encuesta de periodicidad mensual que compila información a nivel de hogares sobre características socioeconómicas y variables relativas al mercado laboral. La información disponible compone un corte transversal repetido, con información de 23 departamentos y la capital de Colombia, con datos mensuales desde 2008 hasta 2017, el tamaño de la muestra por año y departamento se puede observar en el Tabla 1. 
Castro Puello, Ariza Estévez y Ardila Delgado: La población inactiva en Colombia...

\section{A. Variables}

El modelo de estimación aplicado toma como determinantes de la inactividad laboral variables como edad, género, nivel educativo, estrato socioeconómico ${ }^{1}$, estado civil, zona urbana/rural y variables como número de personas en el hogar y número de personas menores de 13 años en el hogar ${ }^{2}$.

Adicionalmente, se toma el salario de las personas ocupadas como proxy de las expectativas de ingreso de los individuos. Para esto se promedió el salario por edad, nivel educativo, mes, año, departamento y zona urbano/rural. Las categorías resultantes fueron emparejadas con los individuos que cumplían estas condiciones ${ }^{3}$.

Las expectativas de ingreso asumen que los individuos para formar sus expectativas de cuanto ganarían pueden observar el salario promedio de personas con su nivel educativo, experiencia (aproximado por la edad), y ubicando tanto por la zona como por el departamento y diferencias temporales teniendo en cuenta el año y el mes.

Las variables referentes a estrato socio económico, estado civil, género, si estudia, si reside en zona urbana (casco urbano) y nivel educativo ingresan al modelo como variables dummies. La variable edad se incluyó en nivel y al cuadrado para captar la relación no lineal entre la edad y la probabilidad de estar inactivo. El número de observaciones, media y desviación estándar de las variables del modelo se puede encontrar en la Tabla 2.

El modelo también toma el logaritmo natural de las expectativas de ingreso, el cual permite calcular una semi-elasticidad, incluyéndose,

1 Forma en la que se clasifican los hogares según las condiciones de la vivienda y el entorno o zona en la que esta se encuentra.

2 Las variables sobre independencia son frecuentemente usadas debido a que los individuos se ven obligados a ingresar al mercado laboral para hacerse cargo de aquellas personas que no pueden hacerlo. La decisión sobre cuando una persona depende del país. Para el caso colombiano, el rango de edad considerado dependiente, está ligado a la definición de edad para trabajar (Aldana \& Arango, 2007)

3 Idealmente, se debería diferenciar por sector económico. No obstante, esta información solo estaba disponible para las personas empleadas y no para los desempleados o inactivos. 


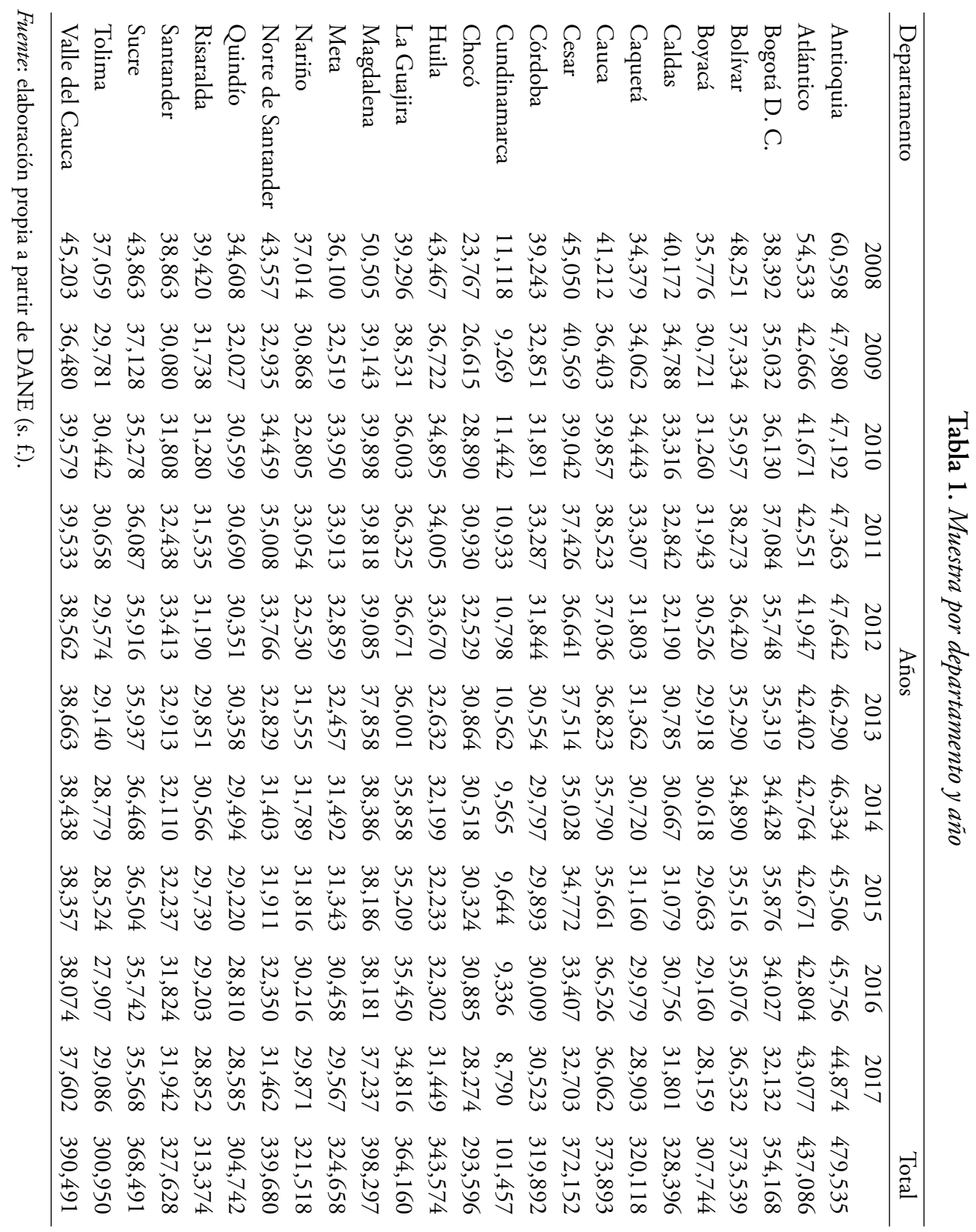


Castro Puello, Ariza Estévez y Ardila Delgado: La población inactiva en Colombia...

Tabla 2. Estadisticas descriptivas (parte 1)

\begin{tabular}{lccc}
\hline Variables & Observaciones & Promedio & Desv. Estándar \\
\hline Inactivo & 6526280 & 0,37 & 0,48 \\
Ingresos (pesos) & 3534253 & 755902 & 980250 \\
Edad & 8021507 & 31 & 21 \\
Es mujer & 8021507 & 0,53 & 0,5 \\
Urbano & 8159139 & 0,9 & 0,3 \\
Número de personas en el hogar & 8026595 & 4 & 2 \\
Personas con menos de 13 años & 8026595 & 1 & 1 \\
\hline
\end{tabular}

Fuente: elaboración propia a partir de DANE (s. f.).

adicionalmente en nivel y de forma cuadrática para captar diferencias no lineales con las expectativas de ingresos tales como una reducción creciente de la probabilidad de ser inactivo con respecto a las expectativas de ingresos. Las variables número de personas en el hogar y número de miembros del hogar menores de 13 años, se involucran al modelo en nivel y en forma cuadrática ${ }^{4}$.

Por último, se calculó para los subgrupos de edad, nivel educativo, mes, año y ubicación del hogar /lugar de residencia (zona urbana-zona rural), la probabilidad de estar empleado como un proxy de las expectativas de conseguir empleo. La variable se incluye en el modelo en nivel y al cuadrado.

Como se puede observar en la Tabla 2, el porcentaje de población inactiva es aproximadamente el $37 \%$, que refleja la media nacional durante el periodo de análisis. El promedio de ingresos de la muestra es de 896 mil pesos colombianos, que es aproximadamente a 1,02 veces el salario mínimo para el último año de la muestra (2017). La edad promedio de los individuos encuestados es de 31 años y el 53\% de la muestra son mujeres. El $90 \%$ de

4 Se incluye el efecto cuadrático para captar cualquier comportamiento no lineal. Se espera que, a mayor número de miembros de un hogar con menos de 13 años, menor probabilidad de estar inactivo. Sin embargo, este efecto se puede incrementar a medida que el numero sube debido a que hay mayor probabilidad de que sea el único miembro en edad de trabajar, o se puede atenuar si el efecto en la participación laboral de la dependencia se activa tan solo con la presencia de un menor de edad. 
las observaciones proviene de personas en zonas urbanas. Los hogares en promedio están conformados por cuatro individuos con un promedio de un menor a 13 años.

En la Tabla 3 podemos observar que el máximo nivel educativo para la mayor parte de la muestra $(30 \%)$ es básica primaria, seguidos por bachilleres que representan el $25 \%$ de la muestra, el 10\% de la muestra está compuesta por población analfabeta y $32 \%$ indica encontrarse estudiando en el momento de ser encuestado.

Tabla 3. Estadisticas descriptivas (Parte 2)

\begin{tabular}{lccc}
\hline Variables & Observaciones & Promedio & Desv. Estándar \\
\hline Sin educación & 7421476 & 0,07 & 0,25 \\
Preescolar & 7421476 & 0,03 & 0,18 \\
Básica Primaria & 7421476 & 0,3 & 0,46 \\
Básica Secundaria & 7421476 & 0,2 & 0,4 \\
Bachiller & 7421476 & 0,25 & 0,43 \\
Técnico o Tecnólogo & 7421476 & 0,07 & 0,25 \\
Universitario & 7421476 & 0,06 & 0,23 \\
Posgrado & 7421476 & 0,02 & 0,14 \\
Analfabeta & 7636785 & 0,1 & 0,31 \\
Estudia & 7636785 & 0,32 & 0,47 \\
\hline
\end{tabular}

Fuente: elaboración propia a partir de DANE (s. f.).

En cuanto al estado civil podemos observar en la Tabla 4 que la muestra está compuesta mayoritariamente por personas solteras $(39 \%)$ en segundo lugar se encuentran las personas en unión libre mayor a 2 años, mientras que el $20 \%$ de la muestra se encuentra casada.

En cuanto a la distribución por estratos socioeconómicos (Tabla 5), tenemos que en la muestra el $70 \%$ de los individuos se encuentra en un estrato bajo, $28 \%$ es de estratos medios y solo el $2 \%$ se catalogaba como estrato alto. 
Castro Puello, Ariza Estévez y Ardila Delgado: La población inactiva en Colombia...

Tabla 4. Estadisticas descriptivas (Parte 3)

\begin{tabular}{lccc}
\hline Variables & Observaciones & Promedio & Desv. Estándar \\
\hline Unión libre menor a 2 años & 6659114 & 0,02 & 0,15 \\
Unión libre mayor a 2 años & 6659114 & 0,22 & 0,42 \\
Casado & 6659114 & 0,2 & 0,4 \\
Separado & 6659114 & 0,11 & 0,31 \\
Viudo & 6659114 & 0,05 & 0,21 \\
Soltero & 6659114 & 0,39 & 0,49 \\
\hline
\end{tabular}

Fuente: elaboración propia a partir de DANE (s. f.).

Tabla 5. Estadisticas descriptivas (Parte 4)

\begin{tabular}{lccc}
\hline Variables & Observaciones & Promedio & Desv. Estándar \\
\hline Estrato Bajo-Bajo & 7773536 & 0,35 & 0,48 \\
Estrato Bajo & 7773536 & 0,35 & 0,48 \\
Estrato Medio-Bajo & 7773536 & 0,21 & 0,41 \\
Estrato Medio & 7773536 & 0,05 & 0,22 \\
Estrato Medio-Alto & 7773536 & 0,02 & 0,12 \\
Estrato Alto & 7773536 & 0,01 & 0,09 \\
Estrato No sabe & 7773536 & 0 & 0,07 \\
Sin estrato & 7773536 & 0,01 & 0,1 \\
\hline
\end{tabular}

Fuente: elaboración propia a partir de DANE (s. f.).

\section{B. Diferencias regionales}

Una explicación a las diferencias que se observan en las tasas de inactividad entre regiones es que estas se deben a diferencias en las características de la población. A continuación, se presenta un análisis exploratorio en donde se comparan y contrastan el conjunto de variables asociadas a parámetros observables y consideradas por el análisis, entre las regiones Caribe, Oriental, Central, Pacífica y Bogotá 5 .

5 La agrupación sigue la usada por el DANE para presentar estadísticas de mercado laboral. 
Los departamentos se agrupan de la siguiente forma:

- Región Caribe: Atlántico, Bolívar, Cesar, Córdoba, Sucre, Magdalena, La Guajira.

- Región Oriental: Norte de Santander, Santander, Boyacá, Cundinamarca, Meta.

- Región Central: Caldas, Risaralda, Quindío, Tolima, Huila, Caquetá, Antioquia.

- Región Pacífica: Chocó, Cauca, Nariño, Valle.

- Bogotá D.C.

Analizando la tasa de inactividad por regiones (Figura 3) se identifica que Caribe reporta la más alta tasa, seguida de la región Central y Pacífica. Bogotá y la región Oriental, por su parte, son las que evidencian la tasa de inactividad más baja.

Figura 3. Inactividad por regiones

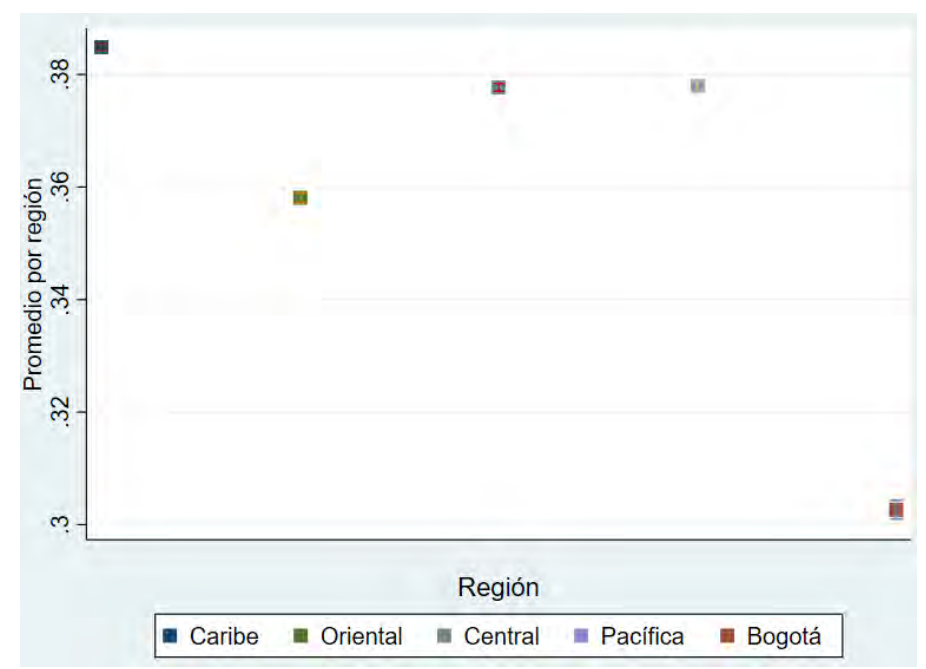

Fuente: elaboración propia a partir de DANE (s. f.).

Estas diferencias en las tasas regionales de inactividad pueden estar relacionado con características observables de la población, En la Figura 4 
se logra identificar como el porcentaje de mujeres es mayor para las regiones central y pacífica. Mientras que la región oriental, caribe y la ciudad de Bogotá poseen porcentajes menores y similares.

Figura 4. Porcentaje de mujeres por región

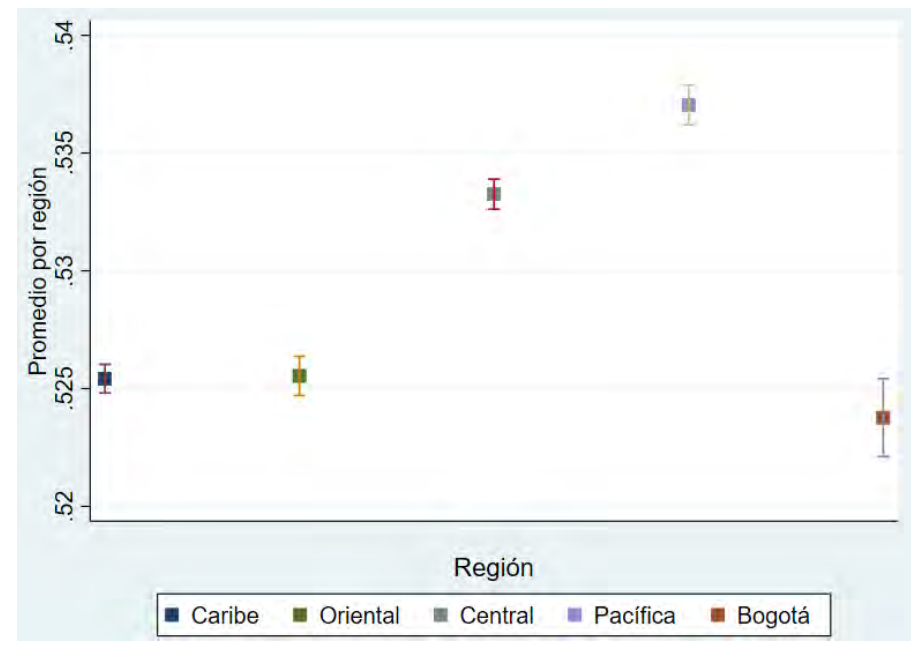

Fuente: elaboración propia a partir de DANE (s. f.).

Como se puede observar en la Figura 5, la región Caribe presenta una población con una edad promedio más baja que la del resto de las regiones colombianas. Por su parte, la región Central reporta la población con la mayor edad promedio.

En cuanto expectativas de ingresos (Figura 6), las cinco regiones analizadas reportan promedios similares, con un valor ligeramente superior en este indicador para la región Oriental y Central, siendo el caso de Bogotá el que reporta el mayor valor.

El porcentaje de personas que se encuentran estudiando está relacionado directamente con la edad de la población, así al igual que en la Figura 5, en la Figura 7 se presenta que en las regiones con menor edad se encuentra mayor porcentaje de estudiantes. 
Figura 5. Edad promedio por región

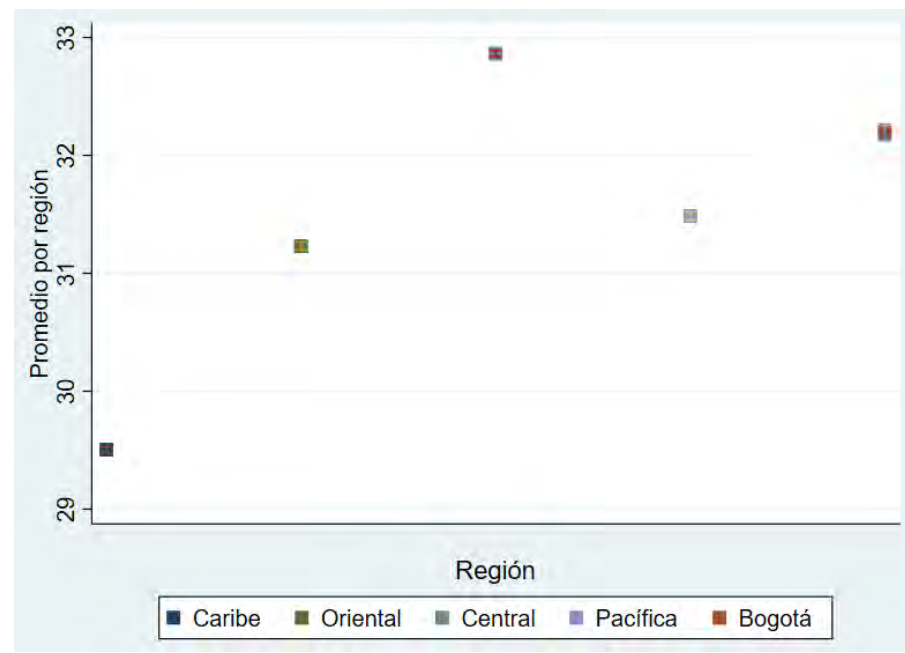

Fuente: elaboración propia a partir de DANE (s. f.).

Figura 6. Expectativas de ingreso promedio por región

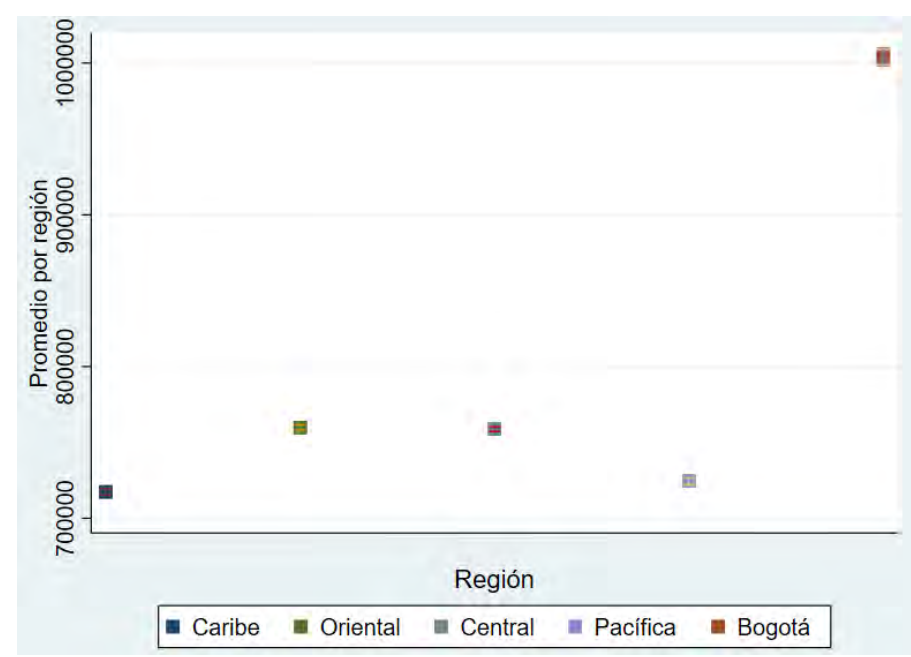

Fuente: elaboración propia a partir de DANE (s. f.). 
Castro Puello, Ariza Estévez y Ardila Delgado: La población inactiva en Colombia...

Figura 7. Porcentaje de población que estudia actualmente por región

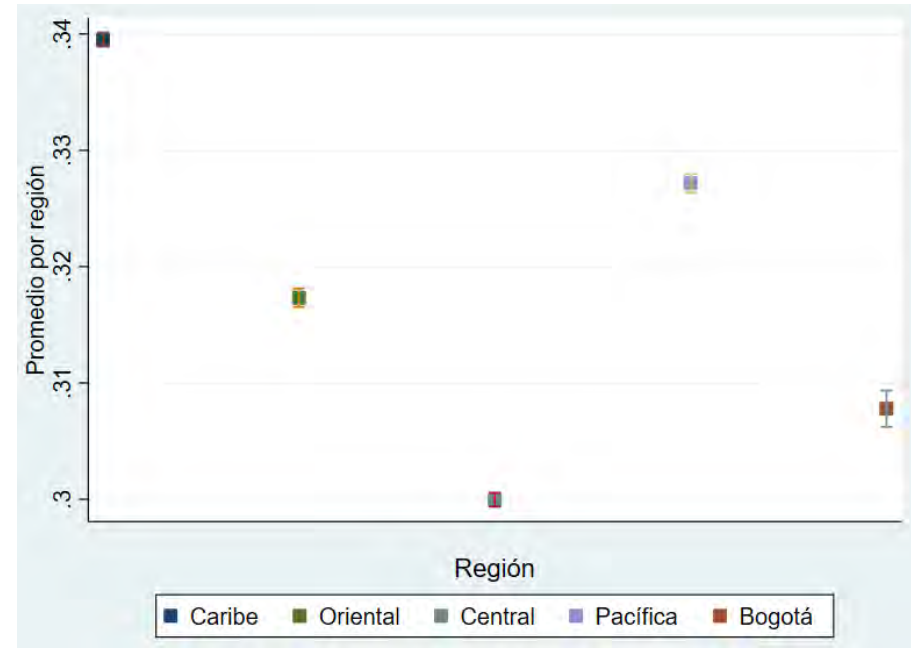

Fuente: elaboración propia a partir de DANE (s. f.).

Figura 8. Porcentaje de población en zona urbana (cabecera municipal)

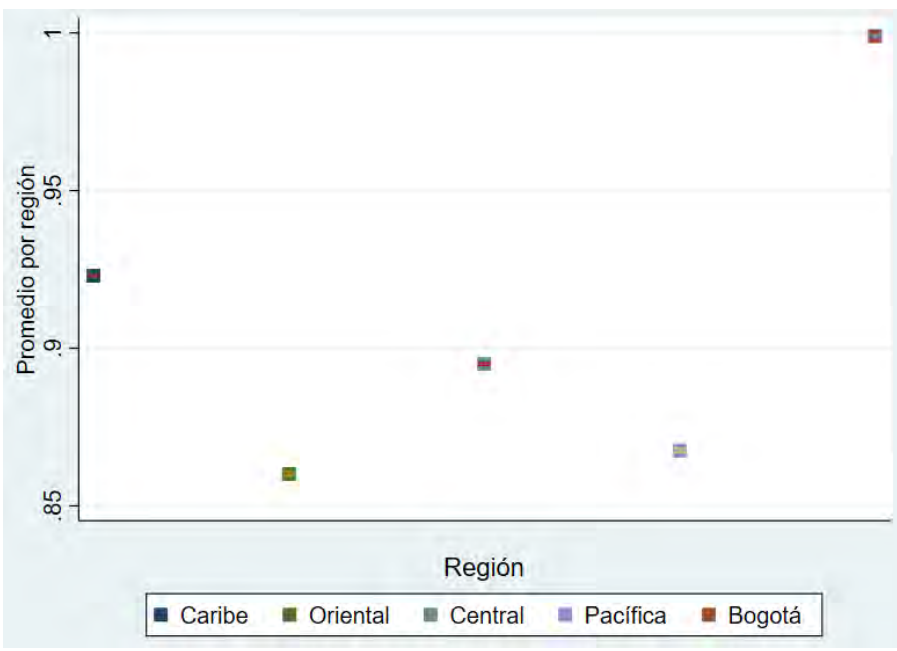

Fuente: elaboración propia a partir de DANE (s. f.). 
Figura 9. Promedio de personas por hogar entre regiones

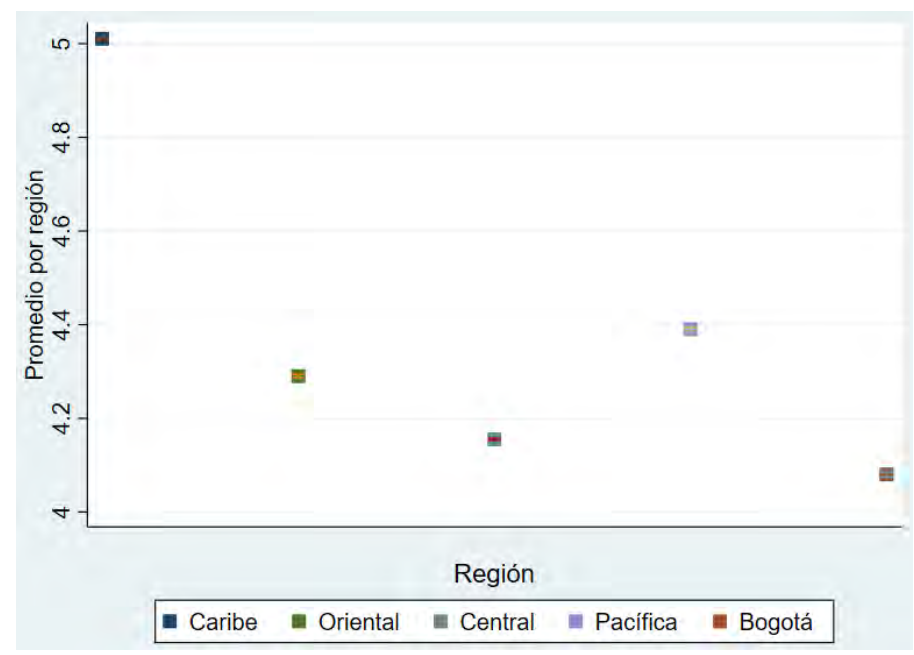

Fuente: elaboración propia a partir de DANE (s. f.).

Excluyendo a la ciudad de Bogotá la región caribe presenta el mayor porcentaje de población ubicada en zonas urbanas seguido por la región central.

La región Caribe es la que tiene el tamaño de hogar más alto con un promedio de cinco personas por hogar mientras que las otras regiones y Bogotá presentan promedios cercanos a cuatro personas.

Observando el número de miembros del hogar con menos de 13 años (Figura 10) tiene un comportamiento similar al del tamaño del hogar de la Figura 9.

En cuanto al analfabetismo (Figura 11) se encuentra que la región caribe tiene el mayor porcentaje entre las regiones analizadas y en contraste la ciudad de Bogotá es el que posee la menor proporción de población analfabeta.

Respecto al nivel educativo, se puede observar en la Tabla 6 un comportamiento similar entre la región Oriental, Central y Pacífica, en el caso de la ciudad de Bogotá para los niveles educativos de bachiller en adelante hay un mayor porcentaje de personas comparando con las otras regiones. Mientras que en el caso de la región Caribe el comportamiento es el contrario 
Castro Puello, Ariza Estévez y Ardila Delgado: La población inactiva en Colombia...

mostrando que para niveles educativos menores a bachiller se presenta una mayor concentración de la población.

Figura 10. Número de miembros del hogar con menos de 13 años por región

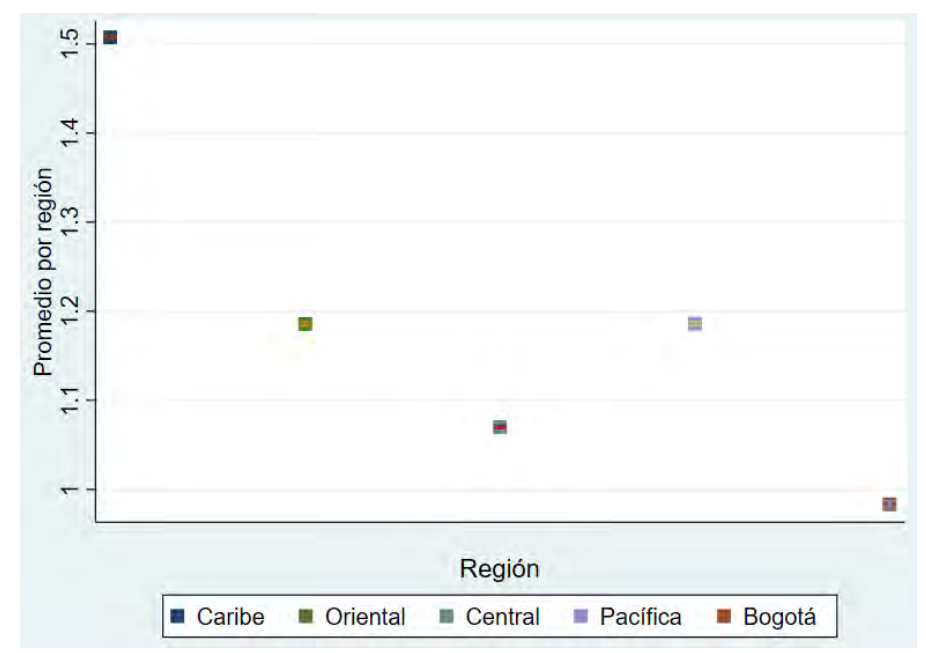

Fuente: elaboración propia a partir de DANE (s. f.).

Tabla 6. Porcentaje de población distribuido por nivel educativo y región

\begin{tabular}{lccccc}
\hline Variables & Caribe & Oriental & Central & Pacífica & Bogotá \\
\hline Sin educación & $7 \%$ & $6 \%$ & $6 \%$ & $6 \%$ & $3 \%$ \\
Preescolar & $5 \%$ & $3 \%$ & $3 \%$ & $3 \%$ & $4 \%$ \\
Básica Primaria & $28 \%$ & $33 \%$ & $32 \%$ & $31 \%$ & $24 \%$ \\
Básica Secundaria & $21 \%$ & $20 \%$ & $21 \%$ & $19 \%$ & $20 \%$ \\
Bachiller & $25 \%$ & $25 \%$ & $24 \%$ & $26 \%$ & $29 \%$ \\
Técnico o Tecnólogo & $7 \%$ & $5 \%$ & $7 \%$ & $6 \%$ & $9 \%$ \\
Universitario & $6 \%$ & $5 \%$ & $5 \%$ & $6 \%$ & $8 \%$ \\
Posgrado & $2 \%$ & $2 \%$ & $2 \%$ & $2 \%$ & $4 \%$ \\
\hline
\end{tabular}

Fuente: elaboración propia a partir de DANE (s. f.). 
Figura 11. Porcentaje de población analfabeta por región

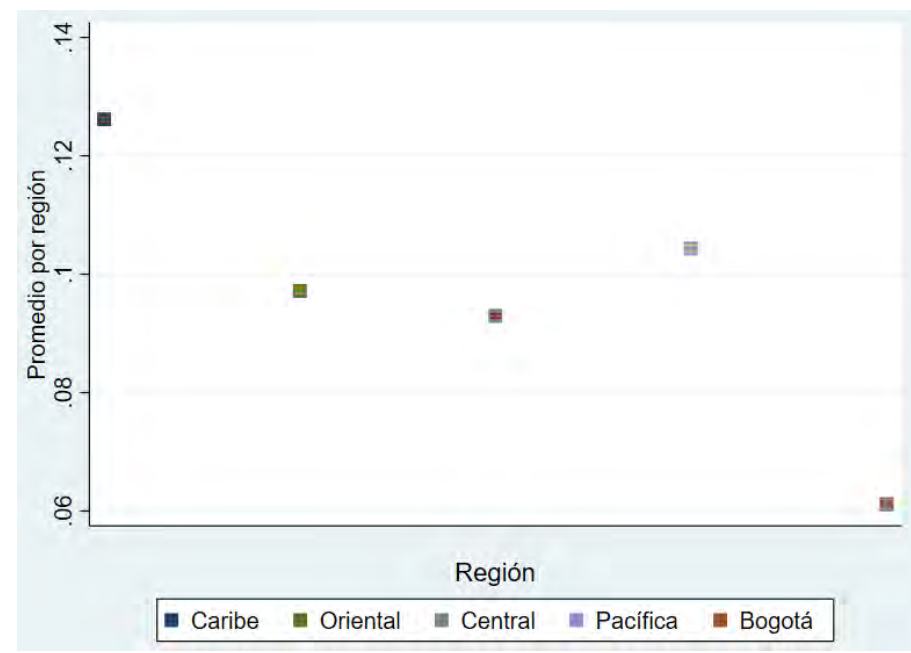

Fuente: elaboración propia a partir de DANE (s. f.).

Con relación al estado civil se identifican similitudes entre todas las regiones con excepción de la región caribe en el que existe un mayor porcentaje de personas en unión libre mayor a 2 años y menor porcentaje de población casada y soltera.

Tabla 7. Porcentaje de población distribuido por estado civil y región

\begin{tabular}{lccccc}
\hline Variables & Caribe & Oriental & Central & Pacífica & Bogotá \\
\hline Unión libre menor a 2 años & $2 \%$ & $2 \%$ & $2 \%$ & $2 \%$ & $3 \%$ \\
Unión libre mayor a 2 años & $27 \%$ & $20 \%$ & $19 \%$ & $22 \%$ & $20 \%$ \\
Casado & $18 \%$ & $24 \%$ & $22 \%$ & $19 \%$ & $21 \%$ \\
Separado & $12 \%$ & $10 \%$ & $11 \%$ & $10 \%$ & $11 \%$ \\
Viudo & $4 \%$ & $5 \%$ & $5 \%$ & $5 \%$ & $4 \%$ \\
Soltero & $38 \%$ & $39 \%$ & $39 \%$ & $42 \%$ & $40 \%$ \\
\hline
\end{tabular}

Fuente: elaboración propia a partir de DANE (s. f.). 
En cuanto a la distribución de población teniendo en cuenta el estrato socioeconómico se evidencia diferencias en la estructura para la mayoría de las regiones, en el caso de la región Caribe y la región Pacífica poseen un mayor porcentaje de población en estratos bajo-bajo, en el caso de Bogotá el comportamiento es similar a la región Oriental y Central con diferencia en que en la ciudad de Bogotá hay menor proporción en estrato bajo-bajo y mayor en medio-bajo.

Tabla 8. Porcentaje de población distribuido por estrato y región

\begin{tabular}{lccccc}
\hline Variables & Caribe & Oriental & Central & Pacífica & Bogotá \\
\hline Sin estrato & $2 \%$ & $1 \%$ & $0 \%$ & $1 \%$ & $0 \%$ \\
Estrato Bajo-Bajo & $49 \%$ & $20 \%$ & $27 \%$ & $42 \%$ & $12 \%$ \\
Estrato Bajo & $28 \%$ & $45 \%$ & $41 \%$ & $29 \%$ & $43 \%$ \\
Estrato Medio-Bajo & $15 \%$ & $26 \%$ & $23 \%$ & $20 \%$ & $35 \%$ \\
Estrato Medio & $4 \%$ & $7 \%$ & $5 \%$ & $4 \%$ & $7 \%$ \\
Estrato Medio-Alto & $1 \%$ & $1 \%$ & $2 \%$ & $2 \%$ & $2 \%$ \\
Estrato Alto & $1 \%$ & $0 \%$ & $1 \%$ & $0 \%$ & $2 \%$ \\
Estrato No sabe & $0 \%$ & $0 \%$ & $0 \%$ & $1 \%$ & $0 \%$ \\
\hline
\end{tabular}

Fuente: elaboración propia a partir de DANE (s. f.).

\section{Metodología}

Luego de identificar que regionalmente se reportan diferencias observables en las características, es necesario realizar un análisis para estimar cómo responde cada población a estas.

Siguiendo a Aldana y Arango (2007), se utilizará un modelo Probit para calcular la probabilidad de ser inactivo de cada individuo a partir de la estimación de tener un salario de reserva $\left(w_{i, t}^{*}\right)$ mayor al salario ofrecido $\left(w_{i, t}\right)$.

$$
P\left(Y_{i, t}=1\right)=P\left(w_{i, t}<w_{i, t}^{*}\right) .
$$

Se asume a su vez que estos salarios están en función de unas variables generales — que afectan a toda la población de igual forma— más un error idiosincrático, es decir un error individual. 


$$
P\left(w_{i, t}<w_{i, t}^{*}\right)=P\left(\bar{w}+e_{i, t}<\bar{w}^{*}+e_{i, t}^{*}\right),
$$

donde $\bar{w}$ y $\bar{w}^{*}$ representan el salario promedio ofrecido y el de reserva y $e_{i, t}$ y $e_{i, t}^{*}$ son los errores idiosincráticos respectivos. Cada salario está en función de unas variables, luego se puede desagregar de la siguiente forma:

$$
\begin{aligned}
& P\left(\bar{w}+e_{i, t}<\bar{w}^{*}+e_{i, t}^{*}\right)= \\
& P\left(\beta_{0}+\sum_{j=1}^{n} X_{j, i, t} \beta_{j}+e_{i, t}<\beta_{0}^{*}+\sum_{j=1}^{m} X_{j, i, t} \beta_{j}^{*}+e_{i, t}^{*}\right),
\end{aligned}
$$

donde $\bar{w}=\beta_{0}+\sum_{j=1}^{n} X_{j, i, t} \beta_{j}$ y $\bar{w}^{*}=\beta_{0}^{*}+\sum_{j=1}^{m} X_{j, i, t} \beta_{j}^{*}$. De esta ecuación, y normalizando por la varianza de $e_{i, t}^{*}$, tenemos:

$$
\begin{array}{r}
P\left(\beta_{0}+\sum_{j=1}^{n} X_{j, i, t} \beta_{j}+e_{i, t}<\beta_{0}^{*}+\sum_{j=1}^{m} X_{j, i, t} \beta_{j}^{*}+e_{i, t}^{*}\right)= \\
P\left(\frac{\beta_{0}-\beta_{0}^{*}+\sum_{j=1}^{n} X_{j, i, t} \beta_{j}-\sum_{j=1}^{m} X_{j, i, t} \beta_{j}^{*}}{\sigma}<\frac{e_{i, t}^{*}-e_{i, t}}{\sigma}\right) .
\end{array}
$$

Si asumimos $\frac{e_{i, t}^{*}-e_{i, t}}{\sigma}=\tau$, tenemos que esta nueva variable al estar conformada por dos normales, la distribución resultante es también normal con parámetros $N\left(0, \sigma_{\tau}\right)$. Por lo anterior, el modelo se describe de la siguiente forma:

$$
P\left(Y_{i, t}=1\right)=1-\Phi\left(X_{i, t}, \beta\right),
$$

donde $Y_{i, t}$ es una variable categórica que refleja la decisión de participar de un individuo $i$, en el periodo $t$ indicando el año. Se asume una forma funcional del error tipo normal señalado como $\Phi$ que depende de dos factores. Por un lado, tenemos las características del individuo $X_{i}$ que influyen en el cálculo del salario de reserva o en el salario de mercado y los parámetros $\beta$, que reflejan la relación entre las características $X_{i, t}$ y el salario de reserva o salario del mercado.

Se estima el modelo anterior para cada departamento para tener una aproximación de los coeficientes de cada variable. Lo que refleja las 
características culturales y tradicionales que influyen en cómo entre regiones se responde de maneras diferentes a los mismos estímulos.

A partir de estas estimaciones y de las diferencias observables de la sección anterior se busca entender las diferencias estructurales de la población inactiva entre departamentos. Para esto se plantea una descomposición entre los determinantes y los parámetros del modelo. Este procedimiento toma dos poblaciones en comparación y separa las diferencias, producto de las características $X_{i, t}$ y diferencias en los parámetros $\beta$.

La diferencia $(D)$ estimada se puede separar de la siguiente forma:

$$
D=\left[E_{\beta_{b}}\left(Y_{a} \mid X_{a}\right)-E_{\beta_{b}}\left(Y_{b} \mid X_{b}\right)\right]+\left[E_{\beta_{a}}\left(Y_{a} \mid X_{a}\right)-E_{\beta_{b}}\left(Y_{a} \mid X_{a}\right)\right],
$$

Donde $a$ indica la muestra del departamento que se evalúa, y $b$ el resto del país. $E_{\beta_{a}}\left(Y_{a} \mid X_{a}\right)$ y $E_{\beta_{b}}\left(Y_{b} \mid X_{b}\right)$ son los valores esperados de inactividad en las muestras $a$ y $b$ respectivamente. En cuanto a $E_{\beta_{b}}\left(Y_{a} \mid X_{a}\right)$ representan el valor esperado de inactividad para la muestra $a$ pero con los parámetros estimados de la muestra $b$.

Esta metodología adapta la descomposición a la Blinder-Oaxaca a modelos no lineales, debido a que la probabilidad de estar inactivo depende del punto de referencia en donde se encuentren los valores de las variables exógenas, por tal motivo la aplicación de modelos lineales no es adecuado para calcular el diferencial entre los grupos de comparación. Esta metodología condiciona a los valores particulares de $X$ de cada subgrupo para estimar cuánto del diferencial es atribuible a cada categoría, es decir, cuánto a las diferencias en $X$ y cuánto a diferencias entre los $\beta$ (Sinning et al., 2008).

\section{Estimación}

El modelo se limitó a las observaciones con datos disponibles ${ }^{6}$ de cada una de las variables seleccionadas ${ }^{7}$. También se eliminaron valores atípicos en

6 Las variables de expectativas de ingreso y expectativa de empleabilidad redujeron significativamente la muestra, particularmente en población menor de edad.

7 Otras variables consideradas pero que no disponían de suficientes datos fueron educación de padre y madre, sector económico de la población inactiva y si el individuo dejó de consumir un alimento en la última semana. 
ingresos, número de personas en el hogar y edad; se eliminó la población que no conocían su estrato o se encontraban en estrato 0. Por último, se eliminaron los registros del mes de enero de 2008 debido a un número inusual de observaciones.

Las variables categóricas usadas de base corresponden a las categorías más comunes de la muestra. Las variables de nivel educativo tienen como base a las personas bachilleres, en estado civil el punto de comparación fue la población soltera y en estrato socioeconómico se encuentra la población de estrato 2. En cuando al departamento de procedencia se toma como base la ciudad de Bogotá al reportar la tasa de inactividad más baja entre las regiones analizadas, como se observó en la Figura 3. Para verificar que el modelo este bien especificado, se realiza una estimación para toda Colombia que se presenta en la Tabla 9.

Tabla 9. Estimación del modelo de regresión para Colombia

\begin{tabular}{|c|c|c|}
\hline Variables & & (1) \\
\hline \multirow[b]{2}{*}{ Expectativa de ingresos } & Nivel & $\begin{array}{c}-3,142^{* * *} \\
(0,0528)\end{array}$ \\
\hline & Cuadrado & $\begin{array}{c}0,105^{* * *} \\
(0,00205)\end{array}$ \\
\hline \multirow[t]{2}{*}{ Expectativa de empleabilidad } & Nivel & $\begin{array}{l}-0,0198^{* * *} \\
(0,000456)\end{array}$ \\
\hline & Cuadrado & $\begin{array}{c}0,000145^{* * *} \\
(2,98 \mathrm{e}-06)\end{array}$ \\
\hline \multirow[t]{5}{*}{ Edad } & Nivel & $\begin{array}{c}-0,131^{* * *} \\
(0,000482)\end{array}$ \\
\hline & Cuadrado & $\begin{array}{c}0,00162^{* * *} \\
(5,83 \mathrm{e}-06)\end{array}$ \\
\hline & Ninguno & $\begin{array}{c}-0,507^{* * *} \\
(0,0164)\end{array}$ \\
\hline & Básica Primaria & $\begin{array}{l}0,0519^{* * *} \\
(0,00329)\end{array}$ \\
\hline & Básica Secundaria & $0,0344^{* * *}$ \\
\hline
\end{tabular}


Castro Puello, Ariza Estévez y Ardila Delgado: La población inactiva en Colombia...

Tabla 9. Continúa

\begin{tabular}{|c|c|c|}
\hline Variables & & (1) \\
\hline \multirow{7}{*}{ Nivel educativo } & & $(0,00299)$ \\
\hline & \multirow{2}{*}{ Técnico o Tecnológico } & $-0,550^{* * *}$ \\
\hline & & $(0,00366)$ \\
\hline & \multirow{2}{*}{ Universitario } & $-0,952^{* * *}$ \\
\hline & & $(0,00638)$ \\
\hline & \multirow{2}{*}{ Posgrado } & $-1,881^{* * *}$ \\
\hline & & $(0,0267)$ \\
\hline \multirow{8}{*}{ Dummies } & \multirow{2}{*}{ Es analfabeta } & $0,205^{* * *}$ \\
\hline & & $(0,0139)$ \\
\hline & \multirow{2}{*}{ Estudia } & $0,843^{* * *}$ \\
\hline & & $(0,00272)$ \\
\hline & \multirow{2}{*}{ Es mujer } & $0,713^{* * *}$ \\
\hline & & $(0,00224)$ \\
\hline & \multirow{2}{*}{ Urbano (Vive en Cabecera) } & $0,565^{* * *}$ \\
\hline & & $(0,00664)$ \\
\hline \multirow{10}{*}{ Estado Civil } & \multirow{2}{*}{ Unión libre menor a 2 años } & $-0,112^{* * *}$ \\
\hline & & $(0,00604)$ \\
\hline & \multirow{2}{*}{ Unión libre mayor a 2 años } & $0,101^{* * *}$ \\
\hline & & $(0,00309)$ \\
\hline & \multirow{2}{*}{ Casado } & $0,284^{* * *}$ \\
\hline & & $(0,00335)$ \\
\hline & \multirow{2}{*}{ Separado } & $-0,307^{* * *}$ \\
\hline & & $(0,00398)$ \\
\hline & \multirow{2}{*}{ Viudo } & 0,00661 \\
\hline & & $(0,00707)$ \\
\hline \multirow{8}{*}{ Estrato socioeconómico } & \multirow{2}{*}{ Estrato 1} & $-0,0631^{* * *}$ \\
\hline & & $(0,00250)$ \\
\hline & \multirow{2}{*}{ Estrato 3} & $0,0831^{* * *}$ \\
\hline & & $(0,00254)$ \\
\hline & \multirow{2}{*}{ Estrato 4} & $0,220^{* * *}$ \\
\hline & & $(0,00460)$ \\
\hline & \multirow{2}{*}{ Estrato 5} & $0,238^{* * *}$ \\
\hline & & $(0,00805)$ \\
\hline
\end{tabular}


Tabla 9. Continúa

\begin{tabular}{|c|c|c|}
\hline \multirow[t]{2}{*}{ Variables } & & (1) \\
\hline & Estrato 6 & $\begin{array}{l}0,195^{* * *} \\
(0,0117)\end{array}$ \\
\hline \multirow{4}{*}{ Número de niños menores de 13} & Nivel & $\begin{array}{c}-0,0272^{* * *} \\
(2,38 \mathrm{e}-05)\end{array}$ \\
\hline & Cuadrado & $\begin{array}{l}0,0046^{* * *} \\
(6,36 \mathrm{e}-08)\end{array}$ \\
\hline & Personas en el hogar & $\begin{array}{c}0,0206^{* * *} \\
(0,000685)\end{array}$ \\
\hline & Constante & $\begin{array}{c}23,92^{* * *} \\
(0,339)\end{array}$ \\
\hline Departamentos & & $\mathrm{Si}$ \\
\hline Años & & $\mathrm{Si}$ \\
\hline Meses & & $\mathrm{Si}$ \\
\hline Seudo R cuadrado & & 0,2745 \\
\hline Observaciones & & 3009904 \\
\hline
\end{tabular}

Error estándar en paréntesis. ${ }^{* * *} \mathrm{p}<0,01,{ }^{* *} \mathrm{p}<0,05,{ }^{*} \mathrm{p}<0,1$.

Fuente: elaboración propia a partir de DANE (s. f.).

El modelo utilizado predice en un 27,45 \% la situación de inactividad de los individuos estudiados. La dirección de las variables es acorde con esperado. Una mayor expectativa de ingresos de su subgrupo poblacional reduce la probabilidad de estar inactivo — por ejemplo, si un individuo de 30 años observa que personas de su edad con el mismo nivel educativo en su ciudad ganan un salario alto se verá tentado a participar en el mercado laboral y dejar la inactividad-. No obstante, si las expectativas salariales del subgrupo son muy altas la probabilidad de estar inactivo empieza a ser mayor.

Lo anterior puede atribuirse a dos fenómenos. El primero estaría relacionado con una mayor probabilidad de que otro miembro de su hogar reciba una remuneración alta y por ende los ingresos del hogar sean mayores reduciendo la necesidad de ingresar al mercado laboral. Una segunda explicación puede deberse a que, las personas interpreten una remuneración muy elevada como una señal de mayores requisitos para ingresar al mercado 
laboral, por lo que la población buscaría mejorar su formación antes de aspirar a la búsqueda de empleo, lo que podría aumentar la probabilidad estar inactivo.

$\mathrm{Al}$ igual que la variable anterior, las expectativas sobre conseguir empleo tienen un efecto decreciente en la probabilidad de estar inactivo y una tendencia a revertir este efecto para los valores más altos de esta variable, esto se puede deber a que una baja expectativa de empleabilidad desmotiva a los agentes a participar del mercado laboral, por lo que aumentos en esta variable llevan a que los individuos se decidan a trabajar o buscar empleo.

De forma similar ocurre con la edad. Sin embargo, la justificación es diferente. A medida que una persona tiene más edad tiene mayor necesidad de buscar empleo para mantenerse, no obstante, para una edad muy avanzada este efecto se revierte debido a que el individuo puede pensionarse o vivir de la renta de sus activos por lo que aumenta su probabilidad de estar inactivo.

Observando la relación con el nivel educativo, se puede reconocer que a mayor educación mayor probabilidad de participar en el mercado laboral —y menor de ser inactivo- con excepción de la población que indica no tener ninguna educación, en cuyo caso tiene menor probabilidad de ser inactivo. Ser analfabeta, estar estudiando, vivir en la zona urbana — cabecera municipalo ser mujer aumenta la probabilidad de estar inactivo.

En cuanto al estado civil se observa que ser viudo y ser soltero tienen estadísticamente el mismo efecto en la probabilidad de estar inactivo, ser separado es el estado civil que más reduce la probabilidad de estar inactivo, mientras que estar casado es el estado que aumenta más la probabilidad de estar inactivo, esto último debido a que al estar casado es posible que algún miembro de la pareja se dedique a labores del hogar.

A mayor estrato social la probabilidad de estar inactivo es mayor, esto debido a que en estratos bajos se tienen mayores niveles de demandas insatisfechas, condición que les obliga a emplearse.

Sobre la composición del hogar se identifica que entre mayor sea el número de miembros de la familia menores de 13 años, es mayor la probabilidad de estar en el mercado laboral, este efecto se reduce a medida que el número de miembros menores aumenta. Con respecto al número 
de personas, hogares grandes generan mayor probabilidad de ser inactivo laboralmente.

La estimación del modelo agregado nos presenta que los coeficientes estimados son acordes a la dirección prevista para cada variable según lo que otros autores han encontrado para el caso colombiano (Aldana \& Arango, 2007).

Para poder observar diferencias entre regiones se presentan las estimaciones en la Tabla 10 agrupando regionalmente.

Tabla 10. Estimación por regiones

\begin{tabular}{|c|c|c|c|c|c|c|}
\hline Variables & & Caribe & Oriental & Central & Pacífica & Bogotá \\
\hline \multirow{3}{*}{$\begin{array}{l}\text { Expectativa de } \\
\text { ingresos }\end{array}$} & Nivel & $-2,109^{* * *}$ & $-3,338^{* * *}$ & $-4,025^{* * *}$ & $-3,789^{* * *}$ & $-3.053^{* * *}$ \\
\hline & & $(0,0999)$ & $(0,132)$ & $(0,0954)$ & $(0,124)$ & $(0,247)$ \\
\hline & Cuadrado & $\begin{array}{l}0,0671^{* * *} \\
(0,00388)\end{array}$ & $\begin{array}{l}0,111^{* * *} \\
(0,00512)\end{array}$ & $\begin{array}{c}0,138^{* * *} \\
(0,00370)\end{array}$ & $\begin{array}{c}0,132^{* * *} \\
(0,00484)\end{array}$ & $\begin{array}{l}0,0960^{* * *} \\
(0,00956)\end{array}$ \\
\hline \multirow{3}{*}{$\begin{array}{l}\text { Expectativa de } \\
\text { empleabilidad }\end{array}$} & Nivel & $-0,0160^{* * *}$ & $-0,0242^{* * *}$ & $-0,0226^{* * *}$ & $-0,0199^{* * *}$ & $-0,0323^{* * *}$ \\
\hline & & $(0,000859)$ & $(0,00123)$ & $(0,000775)$ & $(0,00105)$ & $(0,00231)$ \\
\hline & Cuadrado & $\begin{array}{c}0,000124^{* * *} \\
(5,61 \mathrm{e}-06)\end{array}$ & $\begin{array}{c}0,000172^{* * *} \\
(7,98 \mathrm{e}-06)\end{array}$ & $\begin{array}{c}0,000168^{* * *} \\
(5,08 \mathrm{e}-06)\end{array}$ & $\begin{array}{c}0,000147^{* * *} \\
(6,95 \mathrm{e}-06)\end{array}$ & $\begin{array}{c}0,000216^{* * *} \\
(1,48 \mathrm{e}-05)\end{array}$ \\
\hline \multirow[t]{2}{*}{ Edad } & Nivel & $\begin{array}{l}-0,138^{* * *} \\
(0,000930)\end{array}$ & $\begin{array}{l}-0,132^{* * *} \\
(0,00120)\end{array}$ & $\begin{array}{l}-0,122^{* * *} \\
(0,000824)\end{array}$ & $\begin{array}{l}-0,127^{* * *} \\
(0,00119)\end{array}$ & $\begin{array}{l}-0,137^{* * *} \\
(0,00214)\end{array}$ \\
\hline & Cuadrado & $\begin{array}{c}0,00167^{* * *} \\
(1,15 \mathrm{e}-05)\end{array}$ & $\begin{array}{c}0,00163^{* * *} \\
(1,45 \mathrm{e}-05)\end{array}$ & $\begin{array}{c}0,00153^{* * *} \\
(9,84 \mathrm{e}-06)\end{array}$ & $\begin{array}{c}0,00158^{* * *} \\
(1,44 \mathrm{e}-05)\end{array}$ & $\begin{array}{c}0,00174^{* * *} \\
(2,57 \mathrm{e}-05)\end{array}$ \\
\hline \multirow[t]{9}{*}{ Nivel educativo } & Ninguno & $\begin{array}{c}-0,349^{* * *} \\
(0,0280)\end{array}$ & $\begin{array}{c}-0,477^{* * *} \\
(0,0444)\end{array}$ & $\begin{array}{c}-0,580^{* * *} \\
(0,0285)\end{array}$ & $\begin{array}{c}-0,568^{* * *} \\
(0,0450)\end{array}$ & $\begin{array}{c}-0,947^{* * *} \\
(0,0964)\end{array}$ \\
\hline & Básica Primaria & $\begin{array}{c}-0,0172^{* * *} \\
(0,00651)\end{array}$ & $\begin{array}{c}0,102^{* * *} \\
(0,00795)\end{array}$ & $\begin{array}{c}0,116^{* * *} \\
(0,00561)\end{array}$ & $\begin{array}{c}0,00581 \\
(0,00814)\end{array}$ & $\begin{array}{c}0,0403^{* * *} \\
(0,0144)\end{array}$ \\
\hline & Básica Secundaria & $\begin{array}{c}-0,0265^{* * *} \\
(0,00575)\end{array}$ & $\begin{array}{l}0,0554^{* * *} \\
(0,00744)\end{array}$ & $\begin{array}{l}0,0944^{* * *} \\
(0,00505)\end{array}$ & $\begin{array}{c}-0,0287^{* * *} \\
(0,00773)\end{array}$ & $\begin{array}{l}0,105^{* * *} \\
(0,0123)\end{array}$ \\
\hline & Técnico o & $-0,507^{* * *}$ & $-0,616^{* * *}$ & $-0,574^{* * *}$ & $-0,577^{* * *}$ & $-0,488^{* * *}$ \\
\hline & Tecnológico & $(0,00635)$ & $(0,0106)$ & $(0,00627)$ & $(0,00982)$ & $(0,0158)$ \\
\hline & Universitario & $\begin{array}{c}-0,902^{* * *} \\
(0,0105)\end{array}$ & $\begin{array}{c}-0,941^{* * *} \\
(0,0162)\end{array}$ & $\begin{array}{c}-1,053^{* * *} \\
(0,0126)\end{array}$ & $\begin{array}{c}-0,998^{* * *} \\
(0,0159)\end{array}$ & $\begin{array}{c}-0,855^{* * *} \\
(0,0306)\end{array}$ \\
\hline & Posgrado & $\begin{array}{c}-1,968^{* * *} \\
(0,0647)\end{array}$ & $\begin{array}{c}-1,810^{* * *} \\
(0,0530)\end{array}$ & $\begin{array}{c}-1,932^{* * *} \\
(0,0473)\end{array}$ & $\begin{array}{c}-2,032^{* * *} \\
(0,0648)\end{array}$ & $\begin{array}{c}-1,542^{* * *} \\
(0,101)\end{array}$ \\
\hline & Es analfabeta & $\begin{array}{l}0,129^{* * *} \\
(0,0242)\end{array}$ & $\begin{array}{l}0,262^{* * *} \\
(0,0393)\end{array}$ & $\begin{array}{l}0,308^{* * *} \\
(0,0238)\end{array}$ & $\begin{array}{l}0,158^{* * *} \\
(0,0355)\end{array}$ & $\begin{array}{l}0,405^{* * *} \\
(0,0703)\end{array}$ \\
\hline & Estudia & $\begin{array}{c}0,832^{* * *} \\
(0,00508)\end{array}$ & $\begin{array}{c}0,936^{* * *} \\
(0,00667)\end{array}$ & $\begin{array}{c}0,798^{* * *} \\
(0,00489)\end{array}$ & $\begin{array}{c}0,905^{* * *} \\
(0,00648)\end{array}$ & $\begin{array}{l}0,759^{* * *} \\
(0,0120)\end{array}$ \\
\hline
\end{tabular}

Lecturas de Economía -Lect. Econ. - No. 96. Medellín, Enero-Junio 2022 
Castro Puello, Ariza Estévez y Ardila Delgado: La población inactiva en Colombia...

Tabla 10. Continúa

\begin{tabular}{|c|c|c|c|c|c|c|}
\hline Variables & & Caribe & Oriental & Central & Pacífica & Bogotá \\
\hline & Es mujer & $\begin{array}{c}0,825^{* * *} \\
(0,00428)\end{array}$ & $\begin{array}{c}0,683^{* * *} \\
(0,00552)\end{array}$ & $\begin{array}{c}0,737^{* * *} \\
(0,00390)\end{array}$ & $\begin{array}{c}0,589^{* * *} \\
(0,00555)\end{array}$ & $\begin{array}{l}0,580^{* * *} \\
(0,00949)\end{array}$ \\
\hline & Urbano (Vive en & $0,632^{* * *}$ & $0,493^{* * *}$ & $0,637^{* * *}$ & $0,509^{* * *}$ & $1,975^{* * *}$ \\
\hline & Cabecera) & $(0,0192)$ & $(0,0124)$ & $(0,0104)$ & $(0,0168)$ & $(0,554)$ \\
\hline \multirow{10}{*}{ Estado Civil } & Unión libre & $-0,0556^{* * *}$ & $-0,0961^{* * *}$ & $-0,131^{* * *}$ & $-0,182^{* * *}$ & $-0,0912^{* * *}$ \\
\hline & menor a 2 años & $(0,0112)$ & $(0,0145)$ & $(0,0107)$ & $(0,0155)$ & $(0,0244)$ \\
\hline & Unión libre & $0,115^{* * *}$ & $0,145^{* * *}$ & $0,0818^{* * *}$ & $0,0338^{* * *}$ & $0,144^{* * *}$ \\
\hline & mayor a 2 años & $(0,00568)$ & $(0,00792)$ & $(0,00548)$ & $(0,00746)$ & $(0,0139)$ \\
\hline & \multirow{2}{*}{ Casado } & $0,243^{* * *}$ & $0,308^{* * *}$ & $0,301^{* * *}$ & $0,232^{* * *}$ & $0,347^{* * *}$ \\
\hline & & $(0,00651)$ & $(0,00844)$ & $(0,00563)$ & $(0,00828)$ & $(0,0151)$ \\
\hline & \multirow{2}{*}{ Separado } & $-0,367^{* * *}$ & $-0,313^{* * *}$ & $-0,269^{* * *}$ & $-0,295^{* * *}$ & $-0,248^{* * *}$ \\
\hline & & $(0,00719)$ & $(0,0107)$ & $(0,00686)$ & $(0,00988)$ & $(0,0182)$ \\
\hline & \multirow{2}{*}{ Viudo } & $-0,122^{* * *}$ & $0,0414^{* *}$ & $0,0530^{* * *}$ & 0,0101 & 0,0407 \\
\hline & & $(0,0144)$ & $(0,0181)$ & $(0,0113)$ & $(0,0178)$ & $(0,0317)$ \\
\hline \multirow{10}{*}{$\begin{array}{l}\text { Estrato } \\
\text { socioeconómico }\end{array}$} & \multirow{2}{*}{ Estrato 1} & $-0,0664^{* * *}$ & $-0,0391^{* * *}$ & $-0,0791^{* * *}$ & $-0,0792^{* * *}$ & 0,0116 \\
\hline & & $(0,00417)$ & $(0,00666)$ & $(0,00470)$ & $(0,00651)$ & $(0,0135)$ \\
\hline & \multirow{2}{*}{ Estrato 3} & $0,0537^{* * *}$ & $0,120^{* * *}$ & $0,0909^{* * *}$ & $0,0874^{* * *}$ & $0,0677^{* * *}$ \\
\hline & & $(0,00534)$ & $(0,00582)$ & $(0,00430)$ & $(0,00641)$ & $(0,00966)$ \\
\hline & \multirow{2}{*}{ Estrato 4} & $0,0783^{* * *}$ & $0,339^{* * *}$ & $0,218^{* * *}$ & $0,197^{* * *}$ & $0,522^{* * *}$ \\
\hline & & $(0,00976)$ & $(0,00981)$ & $(0,00785)$ & $(0,0120)$ & $(0,0199)$ \\
\hline & \multirow{2}{*}{ Estrato 5} & $0,0614^{* * *}$ & $0,435^{* * *}$ & $0,289^{* * *}$ & $0,184^{* * *}$ & $0,580^{* * *}$ \\
\hline & & $(0,0172)$ & $(0,0252)$ & $(0,0121)$ & $(0,0191)$ & $(0,0370)$ \\
\hline & \multirow{2}{*}{ Estrato 6} & $-0,00372$ & $0,420^{* * *}$ & $0,250^{* * *}$ & $0,158^{* * *}$ & $0,557^{* * *}$ \\
\hline & & $(0,0227)$ & $(0,0386)$ & $(0,0173)$ & $(0,0372)$ & $(0,0416)$ \\
\hline \multirow{8}{*}{$\begin{array}{l}\text { Número de niños } \\
\text { menores de } 13\end{array}$} & \multirow{2}{*}{ Nivel } & $-0,00967^{* *}$ & $-0,0242^{* * *}$ & $-0,0255^{* * *}$ & $-0,0575^{* * *}$ & $-0,0306^{* * *}$ \\
\hline & & $(0,00415)$ & $(0,00598)$ & $(0,00433)$ & $(0,00609)$ & $(0,0112)$ \\
\hline & \multirow{2}{*}{ Cuadrado } & $0,00366^{* * *}$ & $0,00506^{* * *}$ & 0,00207* & $0,00761^{* * *}$ & 0,00327 \\
\hline & & $(0,00104)$ & $(0,00163)$ & $(0,00122)$ & $(0,00169)$ & $(0,00333)$ \\
\hline & \multirow{2}{*}{$\begin{array}{l}\text { Personas en el } \\
\text { hogar }\end{array}$} & $0.0166^{* * *}$ & $0,0138^{* * *}$ & $0,0230^{* * *}$ & $0,0275^{* * *}$ & $0,0421^{* * *}$ \\
\hline & & $(0.00119)$ & $(0,00175)$ & $(0,00126)$ & $(0,00170)$ & $(0,00320)$ \\
\hline & \multirow{2}{*}{ Constante } & $17,01^{* * *}$ & $25,56^{* * *}$ & $29,51^{* * *}$ & $28,02^{* * *}$ & $23,51^{* * *}$ \\
\hline & & $(0,643)$ & $(0,851)$ & $(0,614)$ & $(0,798)$ & $(1,690)$ \\
\hline Departamentos & & $\mathrm{Si}$ & $\mathrm{Si}$ & $\mathrm{Si}$ & $\mathrm{Si}$ & Si \\
\hline Años & & $\mathrm{Si}$ & Si & $\mathrm{Si}$ & $\mathrm{Si}$ & $\mathrm{Si}$ \\
\hline Meses & & $\mathrm{Si}$ & $\mathrm{Si}$ & $\mathrm{Si}$ & $\mathrm{Si}$ & $\mathrm{Si}$ \\
\hline Seudo R cuadrado & & 0,2708 & 0,2978 & 0,2682 & 0,2803 & 0,2886 \\
\hline Observaciones & & 868456 & 522072 & 974264 & 468975 & 176137 \\
\hline
\end{tabular}

Error estándar en paréntesis $* * * \mathrm{p}<0,01,{ }^{* *} \mathrm{p}<0,05,{ }^{*} \mathrm{p}<0,1$.

Fuente: elaboración propia a partir de DANE (s. f.). 
Las estimaciones por regiones de la Tabla 10 muestran diferencias en las magnitudes y significancias de ciertas variables por región. En un primer lugar podemos observar como en la región Central y Pacífica son más sensibles a las expectativas de ingreso, en contraste con la región Caribe. Con respecto a la expectativa de estar empleado la región que más reacciona a esta variable es la ciudad de Bogotá seguido por la región oriental, mientras que la región caribe es la zona con la menor reacción.

En cuanto a la edad, la región Central y Pacífica son las que menos reacción reportan ante el cambio en este factor. Al ver los coeficientes de los distintos niveles educativos, se encuentra que el efecto de tener un posgrado es menor en la ciudad de Bogotá, además el tener un nivel educativo de básica primaria y secundaria tiene efectos contrarios entre algunas regiones, mientras que para la región Caribe y la Pacífica el efecto reduce la probabilidad de estar inactivo. En las demás regiones ese nivel educativo aumenta la probabilidad de estar inactivo.

Por otro lado, variables como ser analfabeta en la región Central y la ciudad de Bogotá aumentan significativamente la probabilidad de estar inactivo en comparación con otras regiones como la Caribe y la Pacífica. De forma similar estar estudiando aumenta la probabilidad de estar inactivo, sin embargo, en la región Central y Bogotá el aumento es menor que en las otras regiones analizadas.

Se identifica también que para la región Caribe y la Pacífica el ser mujer implica una mayor probabilidad de estar inactivo, comparativamente con otras regiones. Para el factor residir en zona urbana, las regiones Oriental y Pacífica son las menos sensibles a esta variable; el efecto de Bogotá es significativamente alto debido a que la mayoría de su población se es urbana.

Sobre el estado civil se identifica que en las zonas Pacífica y Central encontrarse en unión libre recientemente — menor a dos años- reduce en mayor medida la probabilidad de estar inactivo en comparación de las otras regiones. En las regiones Caribe, Oriental y en la ciudad de Bogotá, el encontrarse en unión libre por un periodo mayor a dos años aumenta en mayor medida la probabilidad de estar inactivo. De forma similar estar casado aumenta en mayor medida la probabilidad de estar inactivo en las regiones 
Oriental, Central y en la ciudad de Bogotá. En la región Caribe el efecto de estar separado reduce la probabilidad de estar inactivo en mayor magnitud que en las demás regiones. Por último, ser viudo tiene resultados ambiguos, mientras que en la región Caribe esto reduce la probabilidad de estar inactivo; en las regiones Oriental y Central esta condición incrementa la inactividad, en las regiones Pacífica y en la ciudad de Bogotá no es relevante esta variable.

El estrato socioeconómico para las regiones oriental y la ciudad de Bogotá es un factor de alta relevancia en comparación con las otras regiones, por otro lado, la región caribe es la región con menor sensibilidad al estrato. El tamaño del hogar tiene un efecto mayor para personas en la ciudad de Bogotá, mientras que el número de menores de 13 años tiene un mayor efecto en la región pacífica.

\section{Descomposición a nivel departamental}

Tomando la muestra disponible para todas las variables utilizadas en la estimación de la sección anterior, se puede observar en la Figura 12 el diferencial entre cada departamento y el resto de Colombia.

En la Figura 12, las barras azules indican los departamentos con mayor población económicamente activa, se destaca Cundinamarca, Tolima, Bogotá y Santander. En verde se pueden identificar los departamentos con mayor porcentaje de inactividad, donde se destaca Bolívar, Cauca, Caquetá y Chocó.

Distinguiendo entre las características de los individuos y los parámetros, se puede observar que departamentos como Cundinamarca, Risaralda, Huila, Valle del cauca y la ciudad de Bogotá, poseen características de sus habitantes, al igual que parámetros — factores culturales u otras predisposiciones- que los hacen más proclives a participar del mercado laboral. De estos, Valle del Cauca y Risaralda pueden atribuir su diferencial a características individuales, mientras que Cundinamarca, Huila y Bogotá pueden atribuir esto a sus parámetros estimados.

De forma similar se encuentra que Chocó, Caquetá, Boyacá, Cesar y Cauca tienen mayor inactividad tanto por características como por parámetros. Boyacá, Caquetá y Chocó pueden atribuir la mayor parte de esta inactividad a 
características de su población. Los departamentos de Cesar y Cauca atribuyen esta diferencia principalmente a sus parámetros.

Figura 12. Diferencia entre tasas de inactividad departamental contra el resto de Colombia

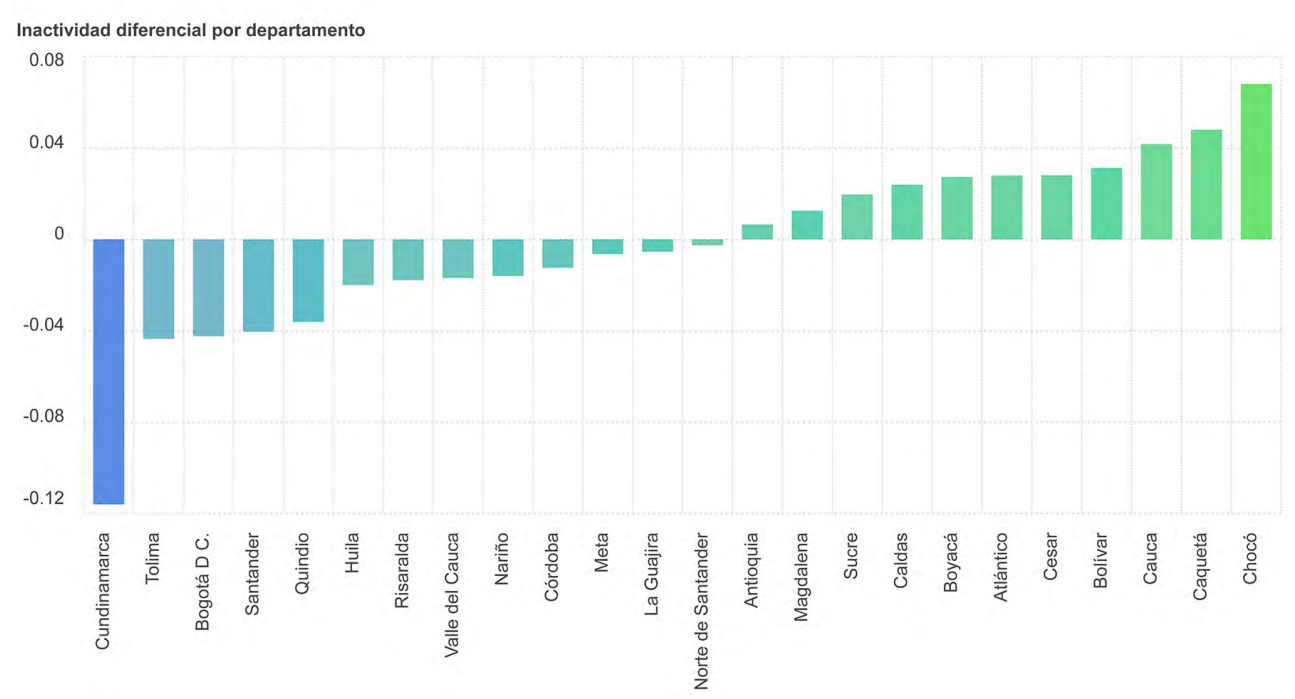

Fuente: elaboración propia a partir de DANE (s. f.).

En los demás departamentos se encuentran presiones ambiguas entre las diferencias producto de parámetros y de sus características. Los departamentos de Quindío y Meta atribuyen su mayor actividad a las características de su población, mientras que, departamentos como Norte de Santander, Guajira, Santander, Córdoba, Tolima y Nariño presentan mayor actividad laboral, producto de sus diferencias en parámetros y características no observables como factores culturales entre otros.

Los departamentos de Bolívar, Magdalena, Caldas, Atlántico y Antioquia tienen mayor inactividad producto de sus factores culturales y parámetros. Sucre es el único de departamento cuya inactividad se genera por las características de población.

Con lo anterior se puede concluir que las diferencias entre características observables y no observables entre los distintos departamentos de 
Colombia no son atribuibles a las mismas variables. Por un lado, la existencia de una mayor proporción de mujeres como en las regiones Central y Pacífica pueden tener efectos diferentes sobre la probabilidad estar inactivo, debido a que en la región Central el ser mujer tiene un coeficiente con mayor magnitud que el de la región Pacífica.

Figura 13. Figura de dispersión entre diferencial en tasas de inactividad atribuido a diferencias en parámetros y diferencial atribuido a diferentes características medias de la población

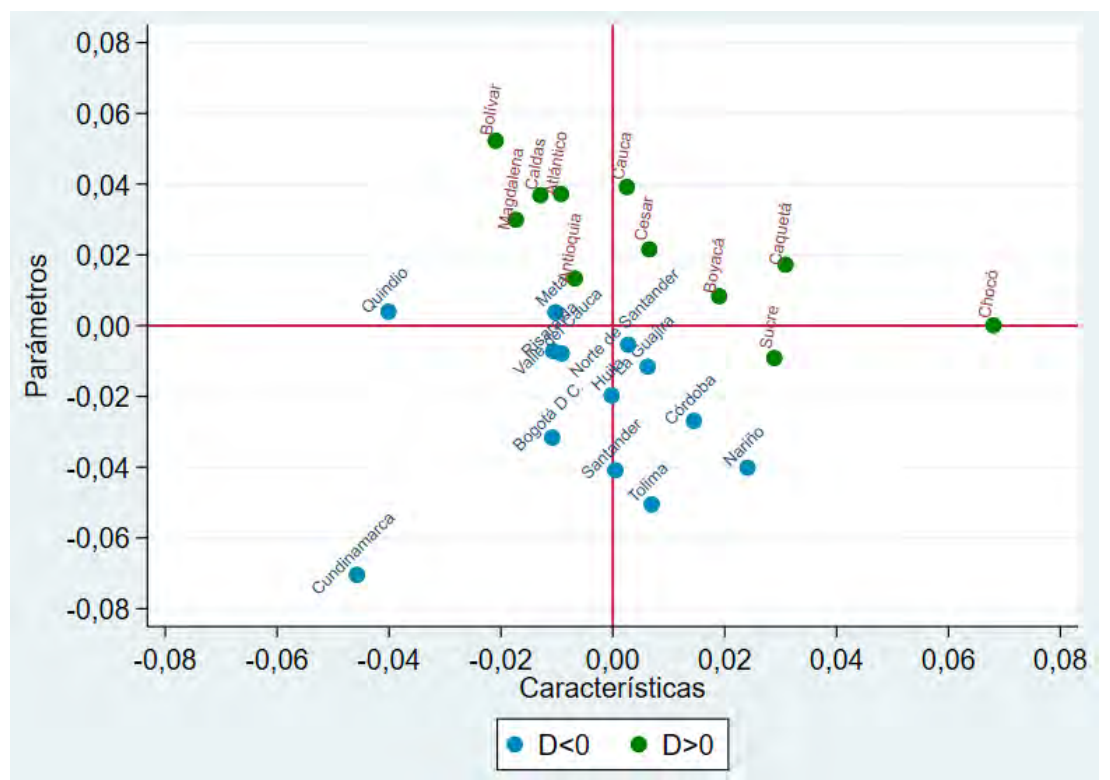

Fuente: elaboración propia a partir de DANE (s. f.).

Otro ejemplo se puede observar entre las regiones Caribe y Pacífica, donde ambas tienen mayor porcentaje de la población estudiando entre las otras regiones. Sin embargo, el efecto de estar estudiando es mayor para la región Pacífica que para la región Caribe. 


\section{Discusión}

Los resultados mostrados deben entenderse como la agregación de múltiples efectos y no se puede atribuir a una variable en particular. Por ejemplo, entre variables culturales se pueden incluir fenómenos como una mayor propensión de la población femenina a realizar labores domésticas en algunas zonas, o una mayor tendencia de la población joven para formarse antes de iniciar su vida laboral. Distinguir a qué factor específico se atribuye, requiere un análisis más profundo.

Otro factor importante para comprender los resultados es relativo a la migración por trabajo. En el caso de Bogotá y Cundinamarca es notable el efecto que tienen sobre sus estadísticas el hecho de que, al ser la capital del país, atraen de todos los demás departamentos personas en búsqueda de empleo. Las zonas aledañas a Bogotá, en especial municipios de Cundinamarca, son objeto de migración de esta índole debido a su cercanía y bajos costos de vida. También, en este sentido se deben considerar complejos productivos como zonas francas, establecimientos mineros y petroleros que atraen mano de obra para su operación —como Barrancabermeja, en el departamento de Santander-.

\section{Conclusiones}

El mercado laboral colombiano posee características estructurales tan variadas como regiones y departamentos. Esta diversidad implica un trabajo particularizado en el planteamiento de políticas públicas sobre empleo. Analizar la tasa global de participación permite tener una mayor comprensión sobre las variaciones del desempleo en cada zona. Poblaciones con bajas tasas de desempleo, pero con altas tasas de inactividad pueden ser señales de trabajadores desmotivados, lo que conllevaría a mayores actividades informales o incluso delincuenciales.

No obstante, se pueden identificar determinantes generales de la tasa de inactividad. En este artículo se encontraron estimaciones que confirman lo encontrado en estudios previos (Aldana \& Arango, 2007) sobre la relación entre variables como edad, género o educación con la probabilidad de estar 
inactivo. Adicionalmente, se encontró que las dos nuevas variables incluidas — expectativa de ingresos y expectativa de empleabilidad — son significantes y con efectos similares sobre la tasa de inactividad, donde para valores bajos de cada una de las variables un incremento reduce la tasa de inactividad, mientras que, en lugares con valores altos, un incremento puede generar un aumento en la inactividad.

Las diferentes regiones del país presentan comportamientos variables no solo en su composición si no también en su respuesta antes las mismas variables. Se encuentra evidencia a favor de que variables como el género puede ser muy importantes para explicar la inactividad en algunas zonas del país. Se evidencian efectos diferenciales del nivel educativo entre regiones, donde, por ejemplo, las personas con básica secundaria tienen menor probabilidad de estar inactivos que un bachiller en las regiones Caribe y Pacífica, mientras que en las demás puede aumentar la inactividad laboral.

En específico, se encuentran diferencias significativas entre regiones, donde se pueden distinguir cuatro grupos de departamentos. En el primer grupo hay tasas de inactividad bajas debido a la composición de su población y a la forma como se comportan. En el segundo, se tiene el comportamiento opuesto, es decir tanto por características observables de sus habitantes como por las no observables tienen una mayor tasa de inactividad. Y en el tercer y cuarto grupo hay comportamientos ambiguos, donde la predisposición cultural va en contra de las características de su población y viceversa.

Por último, distinguir entre diferencias por características de la población y diferencias en factores culturales, nos da una herramienta adicional para conocer qué zonas del país tienen un mercado laboral más sensible a - por ejemplo- cambios en la pirámide poblacional, mayor cobertura en educación, cambios en sistemas pensionales o variaciones en el salario medio - cambios en características - o por el contrario, qué zonas son culturalmente más sensibles o no a los distintos determinantes de la participación laboral. Dado lo anterior, queda abierta la discusión de uso de políticas de empleabilidad particularizadas para ciertas zonas del país, debido a que una política que promueva la participación laboral podría generar un incremento mayor en las áreas más proclives, generando mayor desempleo entre otros factores. 


\section{Referencias}

Aldana, D., \& Arango-Thomas, L. E. (2007). Participación laboral en Ibagué. Borradores de Economía (439), 1-31. https://www.banrep.gov.co/docu $\mathrm{m} / \mathrm{ftp} /$ borra439.pdf

Benitez-Silva, H. (2000). Micro Determinants of Labor Force Status Among Older Americans [Working Papers 00-07, Stony Brook University, Department of Economics]. https://www.stonybrook.edu/commc ms/economics/research/papers/2000/00-07.pdf

Blinder, A. (1973). Wage Discrimination Reduced Form and Structural Estimates. Journal of Human Resources 8(4), 436-455. https://doi.or $\mathrm{g} / 10.2307 / 144855$

Dagsvik, J. K., Kornstad, T., \& Skjerpen, T. (2013). Labor Force Participation and the Discouraged Worker Effect. Empirical Economics, (45), $401-$ 433. https://doi.org/10.1007/s00181-012-0598-9

Departamente Administrativo Nacional de Estadística (DANE). (s. f.). Gran Encuesta Integrada de Hogares - GEIH. Consultado el 30 de Agosto de 2019. https://www.datos.gov.co/Estad-sticas-Nacionales/Gran-Encu esta-Integrada-de-Hogares-GEIH/mcpt-3dws

Dueñas Fernández, D., Iglesias Fernández, C., \& Llorente Heras, R. (2014). Descomposición del GAP salarial por género en el mercado de trabajo español. V Congreso Universitario Internacional Investigación y Género (pp. 703-722), Sevilla. http://hdl.handle.net/11441/40954

Gunderson, M. (1977). Logit Estimates of Labour Force Participation Based on Census Cross-Tabulations. The Canadian Journal of Economics, 10(3), 453-462. https://doi.org/10.2307/134468

Heckman, J. (1979). Sample Selection Bias as a Specification Error. Econometrica, 47 (1), 153-161. https://doi.org/10.2307/1912352

Karaoglan, D., \& Okten, C. (2015). Labor-Force Participation of Married Women in Turkey: A Study of the Added-Worker Effect and the 
Castro Puello, Ariza Estévez y Ardila Delgado: La población inactiva en Colombia...

Discouraged-Worker Effect. Emerging Markets Finance \& Trade, 51 (1), 274-290. https://doi.org/10.1080/1540496X.2015.1011535

Lara, B., Valdés, G., \& Meller, P. (2011). Female discrimination at the professional level in Chile. Interciencia, 36(11), 823-830. https://www. interciencia.net/wp-content/uploads/2018/01/823-MELLER-8.pdf

López, H. (2001). Características y determinantes de la oferta laboral Colombiana y su relación. En M. Urrutia, Empleo y Economía (p.p. 156192). Banco de la República.

Machado, J. A., \& Mata, J. (2005). Counterfactual Decomposition of Changes in Wage Distributions Using Quantile Regression. Journal of applied econometrics, 20(4), 445-465. https://doi.org/10.1002/jae.788

Meller, P., Valdés, G., \&, Lara, B. (2011). Female Discrimination at the Professional Level in Chile. Interciencia, 36 (11), 823-830. https://ww w.interciencia.net/wp-content/uploads/2018/01/823-MELLER-8.pdf

Mroz, T. (1987). The Sensitivity of an Empirical Model of Married Women's Hours of Work to Economic and Statistical Assumptions. Econometrica, 55(4), 765-799. https://doi.org/0012-9682(198707)55:4<765: TSOAEM $>2.0 . \mathrm{CO} ; 2-\mathrm{S}$

O’Brien, M. (2011). Discouraged Older Male Workers and the Discouraged Worker Effect. Australian Journal of Labour Economics: A Journal of Labour Economics and Labour Relations, 14 (3), 217-235. https://ro .uow.edu.au/commpapers/956/

Oaxaca, R. L. (1973). Male-Female Wage Differentials in Urban Labor Markets. International Economic Review 14 (3), 693-709. https://do i.org/10.2307/2525981

Ospino, C. G., Roldán-Vasquez, P., \& Barraza-Narváez, N. (2010). La descomposición salarial de Oaxaca- Blinder: Métodos, críticas y aplicaciones. Una revisión de la literatura. Revista de Economía del Caribe (5), 237-274. https://rcientificas.uninorte.edu.co/index.p $\mathrm{hp} / \mathrm{economia/article/view/1258}$ 
Santa María, M., \& N., R. (2001). La participación laboral: ¿qué ha pasado $y$ qué podemos esperar? [documento de trabajo 146, Archivos de Economía]. Departamento Nacional de Planeación. https://colabora cion.dnp.gov.co/CDT/Estudios\%20Econmicos/146.pdf

Sinning, M., Hahn, M., \& Bauer, T. (2008). The Blinder-Oaxaca Decomposition Fornonlinear Regression Models. The Stata Journal 8(4), 480-492. https://doi.org/10.1177/1536867X0800800402

Spencer, B. G. (1973). Determinants of the Labour Force Participation of Married Women: A Micro-Study of Toronto Households. The Canadian Journal of Economics, 6(2), 222-238. https://doi.org/10.2307/134192

Tenjo, J., \& Ribero, R. (1998). Participación, desempleo y mercados laborales en Colombia [documento de trabajo 81, Archivos de Economía]. Departamento Nacional de Planeación. https://colaboracion.dnp.g ov.co/CDT/Estudios\%20Econmicos/81.pdf

Uribe, J. (2006). Ensayos de Economía aplicados al mercado laboral. Universidad del Valle. 